\title{
Nanocarrier drug resistant tumor interactions: novel approaches to fight drug resistance in cancer
}

\author{
Aleksandra Benko, ${ }^{1, *}$, David Medina-Cruz ${ }^{2, *}$, Ada Vernet-Crua ${ }^{2}$, Catherine P. O'Connell², Małgorzata \\ Świętek ${ }^{3}$, Hamed Barabadi ${ }^{4}$, Muthupandian Saravanan ${ }^{5}$, Thomas J. Webster ${ }^{2}$ \\ 'AGH University of Science and Technology, Faculty of Materials Science and Ceramics, Krakow 30059, Poland. \\ 2Department of Chemical Engineering, Northeastern University, Boston, MA 02115, USA. \\ ${ }^{3}$ Institute of Macromolecular Chemistry, Czech Academy of Sciences, Prague 16206, Czech Republic. \\ ${ }^{4}$ Department of Pharmaceutical Biotechnology, School of Pharmacy, Shahid Beheshti University of Medical Sciences, Tehran \\ 19919-53381, Iran. \\ ${ }^{5}$ Department of Medical Microbiology and Immunology, Division of Biomedical Sciences, School of Medicine, College of Health \\ Sciences, Mekelle University, Mekelle 231, Ethiopia. \\ *These authors contributed equally to this work.
}

Correspondence to: Dr. Thomas J. Webster, Department of Chemical Engineering, Northeastern University, 216 Cullinane, 360 Huntington Avenue, Boston, MA 02115, USA. E-mail: th.webster@neu.edu

How to cite this article: Benko A, Medina-Cruz D, Vernet-Crua A, O'Connell CP, Świętek M, Barabadi H, Saravanan M, Webster TJ. Nanocarrier drug resistant tumor interactions: novel approaches to fight drug resistance in cancer. Cancer Drug Resist 2021;4:264-97. http://dx.doi.org/10.20517/cdr.2020.81

Received: 11 Sep 2020 First Decision: 26 Oct 2020 Revised: 2 Nov 2020 Accepted: 6 Nov 2020 Available online: 19 Jun 2021

Academic Editor: Godefridus J. Peters Copy Editor: Cai-Hong Wang Production Editor: Jing Yu

\begin{abstract}
Cancer is one of the biggest healthcare concerns in our century, a disease whose treatment has become even more difficult following reports of drug-resistant tumors. When this happens, chemotherapy treatments fail or decrease in efficiency, leading to catastrophic consequences to the patient. This discovery, along with the fact that drug resistance limits the efficacy of current treatments, has led to a new wave of discovery for new methods of treatment. The use of nanomedicine has been widely studied in current years as a way to effectively fight drug resistance in cancer. Research in the area of cancer nanotechnology over the past decades has led to tremendous advancement in the synthesis of tailored nanoparticles with targeting ligands that can successfully attach to chemotherapy-resistant cancer by preferentially accumulating within the tumor region through means of active and passive targeting. Consequently, these approaches can reduce the off-target accumulation of their payload and lead to reduced cytotoxicity and better targeting. This review explores some categories of nanocarriers that have been used in the treatment of drug-resistant cancers, including polymeric, viral, lipid-based, metal-based, carbon-based, and magnetic nanocarriers, opening the door for an exciting field of discovery that holds tremendous promise in the treatment of these tumors.
\end{abstract}

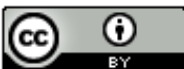

(C) The Author(s) 2021. Open Access This article is licensed under a Creative Commons Attribution 4.0 International License (https://creativecommons.org/licenses/by/4.0/), which permits unrestricted use, sharing, adaptation, distribution and reproduction in any medium or format, for any purpose, even commercially, as long as you give appropriate credit to the original author(s) and the source, provide a link to the Creative Commons license, and indicate if changes were made. 
Keywords: Drug-resistance, cancer, nanotechnology, nanocarriers, drug delivery

\section{DRUG-RESISTANT TUMORS}

Cancer comprises a conjunction of different diseases, which involve the uncontrollable growth of cells generating tumors. Cancerous cells can interfere with various cell processes, such as attachment, division, or motility. Additionally, they can spread into other tissues, forming metastases ${ }^{[1]}$. Nowadays, cancer is one of the major healthcare concerns worldwide. According to the World Health Organization, approximately 10 million people died in 2018 around the globe due to cancer. Moreover, in the US, cancer is the second leading cause of death, with over half a million deaths per year, and the American Cancer Society estimates that there will be approximately 2 million new cases diagnosed by $2020^{[2]}$.

The battle to fight cancer has been present since early human civilization. Reports from the Ancient Greeks and Romans describe basic surgeries to remove breast tumors. However, it was not until the 1940s when chemotherapy treatments were implemented, using drugs to reduce or eliminate cancer cells ${ }^{[3]}$. Since then, a wide variety of treatments combining physics, biology, and molecular genetics have been used to improve recovery rates, such as radiotherapy, hyperthermia, or immunotherapy. Nonetheless, there is still not a definitive cure, and most treatments rely on chemotherapy combined with radiotherapy used to sensitize cancer cells. This increases chemotherapy efficacy while causing increased systemic toxicity and mortality ${ }^{[4,5]}$.

Drug-resistance in cancer is the ability of cancerous cells to become tolerant of the treatments that once could kill them. There are multiple factors responsible for developing resistance; some rely on evolution and spontaneous mutations (intrinsic), others are a consequence of the uptake of drugs (acquired or extrinsic $)^{[6]}$. Over time, leading mechanisms of drug resistance were described as drug inactivation, drug target alteration, drug efflux, reduced drug uptake, cell death inhibition, and DNA damage repair [Figure 1$]^{[7]}$. Approximately $90 \%$ of current chemotherapy treatment failures are related to drug resistance, indicating the seriousness of this issue ${ }^{[8]}$.

Drug inactivation is a consequence of the interaction between the drug and the proteins present in the body, which can affect its structure and/or the molecular mechanisms of the drug. For instance, overexpression of tau, a microtubular-associated protein, was reported to participate in docetaxel (DTX) resistance in various prostate cancer cells ${ }^{[9]}$. Moreover, as some types of anti-cancer drugs are activated upon metabolic digestion, disturbance of this process leads to incomplete activation and acquisition of drug resistance ${ }^{[10]}$. Another mechanism of drug resistance is known as the alteration of drug targets. Modifications and mutations can affect either the drug target or the signaling pathway it controls, which can alter drug efficacy ${ }^{[11]}$. For instance, different mutations in the epidermal growth factor receptor (EGFR) and tyrosine kinase inhibitors (TKI), a target for osimertinib in lung cancer, resulted in an increased drugresistance. Suzawa et al. ${ }^{[12]}$ specifically showed how the amplification in the MET gene could account for up to $22 \%$ of resistance in the EGFR and TKI.

Drug efflux is one of the most studied mechanisms of drug resistance in cancer. The ATP-binding cassette (ABC) family are a group of transport proteins that move compounds such as amino acids, ions, lipids, and drugs through the cell membrane. An alteration or overexpression in the cell transporters can affect the drug export, uptake, and accumulation, making chemotherapy drugs unusable. Specifically, drug efflux is known as the process of drug pumping outside the cell ${ }^{[13]}$. Interestingly, this process may be used to develop resistance against one drug while causing sensitivity towards another. This was proven by Hermawan et al. ${ }^{[14]}$, who found that continuous treatment with salinomycin induced drug resistance against salinomycin, while treatment sensitized drug-resistant cancer cells towards DOX. 


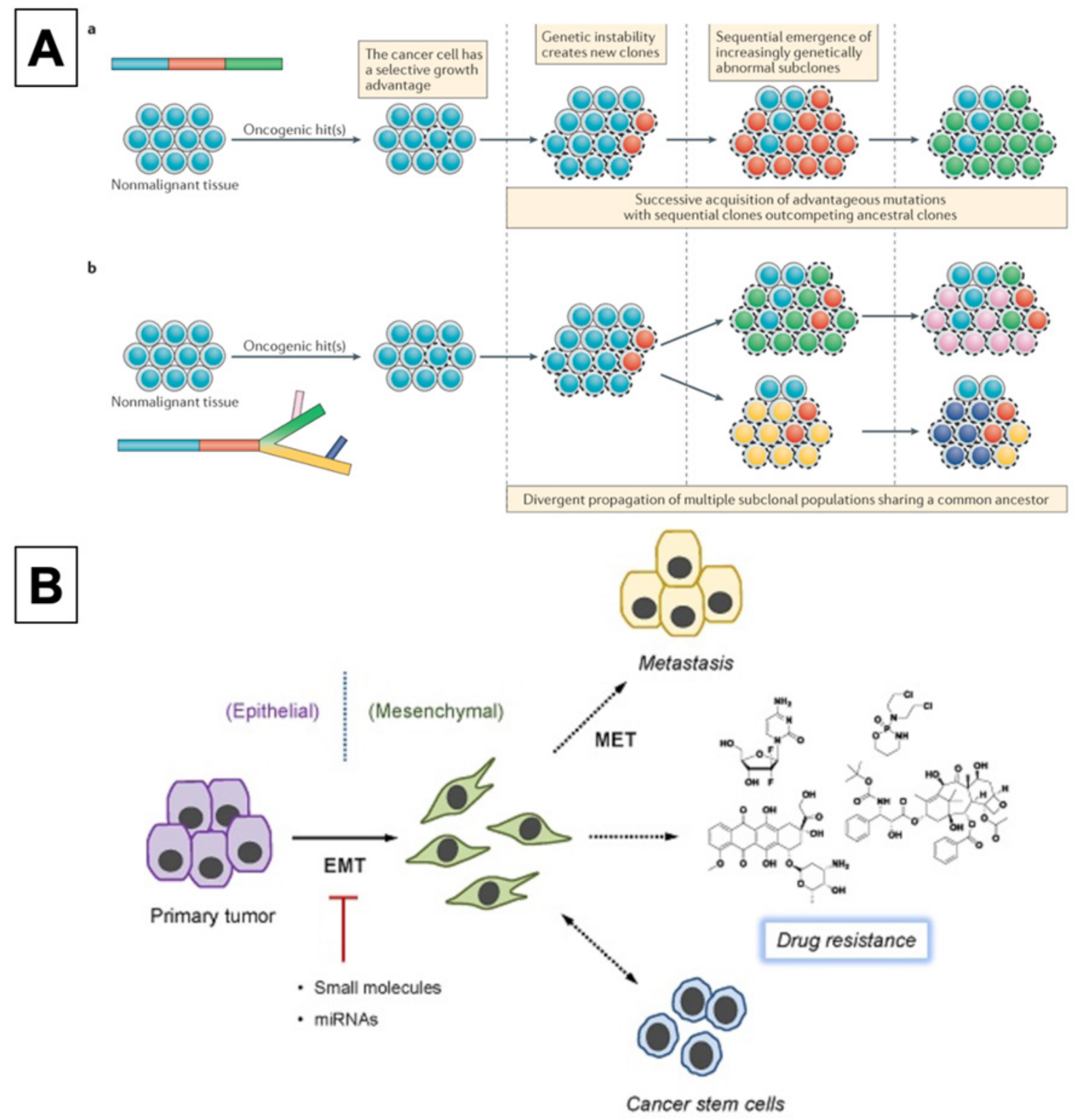

Figure 1. Tumor heterogeneity evolution (a) linear or (b) branched $(A)^{[20]}$; Epithelial-mesenchymal transition mechanism $(B)^{[23]}$

There are two main processes of cell death - autophagy and apoptosis - which are known as programmed necrosis and cell death, respectively. These processes can be modified or inhibited, inducing cancer cell resistance. Apoptosis resistance can be acquired by different pathways, via receptors, like the tumor necrosis factor family or mitochondria. Meanwhile, autophagy is mainly related to hypoxic processes ${ }^{[15]}$. For instance, alterations in the programmed cell death ligand (PD-L1) cause the acquisition of resistance in anaplastic lymphoma kinase proteins of non-small cell lung cancer ${ }^{[16]}$. On the other hand, Toth et al. ${ }^{[17]}$ showed two different resistance pathways for patients with bone-metastatic castration-resistant prostate cancer. In vivo and in vitro modeling demonstrated that resistance to phosphoinositide 3-kinase (PI3K) induced reduced oxidative stress and prevented cell death in a hypoxic microtumor environment.

Moreover, some chemotherapy drugs, especially platinum-based ones, have demonstrated the ability to cause either indirect or direct DNA damage, which can trigger resistance. DNA damage repair systems, 
such as the recombination repair system or the nucleotide excision repair system, are complexes whose principal function is to amend the damage. In cancer cells that develop resistance, those DNA damage repair systems can be inhibited by mutation or gene silencing, leading to further mutations and increasing resistance ${ }^{[18]}$. As described by Nogales et al. ${ }^{[19]}$, the presence of several enzymes such as DNA and RNA helicases in ovarian and lung cancer was related to Pt/carboplatin resistance, and gene silencing was reported to prevent the drug resistance-associated to platinum-derived compounds.

Tumor heterogeneity and microenvironment can also play an important role in drug-resistance. Tumors can have wide variability in terms of phenotype and morphology, such as differential gene expression and motility or metastatic potential. Motility and metastatic potentials are related to epigenetic, transcriptomic, genetic, and proteomic factors such as translocations of RNA or chromosomal rearrangements ${ }^{[20]}$. This variability can be seen in cells coming from the same tumor type; therefore, each cancer cell can react differently when subjected to the same drug. As a consequence, lesion-specific responses were described in some cancer types such as colorectal, when targeted therapies were used ${ }^{[21]}$. Specific extracellular matrix growth factors and stromal cells found in the tumor microenvironment have also been reported to participate in drug-resistant mechanisms as they interfere and modify cell communication ${ }^{[22]}$.

Furthermore, the epithelial-mesenchymal transition (EMT) mechanism is an emerging area of research in terms of cancer drug resistance. EMT is a biological process in which tumors become metastatic. A suppression or modification in EMT can induce changes in cell adhesion and attachment receptors, as well as cell motility and angiogenesis processes ${ }^{[23]}$. EMT, other than promoting the formation of more metastatic cells, can also enhance the survival of those already present, leading to unwanted drug resistance to the treatments ${ }^{[24]}$. As an example, the pre-mRNA processing factor (PRPF), a protein from the kinase family, was found to be overexpressed in some cancers such as prostate and melanoma. In that case, due to arrangements in actin in the cytoskeleton, alterations in cellular morphology occurred. It was demonstrated that PRPF triggers EMT, blocking the apoptotic effects of some drugs like resveratrol, hence triggering drug-resistance in HCT116 colon cancer cells ${ }^{[25]}$. Furthermore, the formation of persister cancer cells (PCC), rare immortal cells that can self-renew through division, is connected with the EMT effect. PCC is in the quiescence state, mitigating traditional therapies that target fast-proliferating cells. These cells are also thought to be responsible for cancer remission and are particularly hard to target ${ }^{[26]}$.

In summary, drug resistance in cancer cells limits the efficacy of current approaches to defeat cancer, especially the late-diagnosed, metastatic ones, decreasing the curability and survival rate and increasing the risk of disease recurrence and relapse. Although several mechanisms such as drug efflux, drug target inactivation, tumor heterogeneities, or DNA damage repair are extensively studied, there are still plenty of unknowns in the field and a large need to develop new treatment strategies. In this review, we will revise and discuss several technologies based on nanomedicine approaches to overcome cancer cell resistance and analyze future challenges that need to be addressed.

\section{NANOCARRIERS FOR THE TREATMENT OF DRUG RESISTANCE IN TUMORS}

Nanomaterials have been extensively investigated for their use in anticancer therapies to improve therapeutic approaches such as drug delivery, increase the efficacy of treatment, reduce side effects, and overcome drug resistance. The use of nanomaterials for drug delivery to tumors has been presented as an alternative to conventional chemotherapy treatments over the past years. An interesting advantage of nanoparticle-assisted drug delivery is the selective targeting to tumors, which might overcome several factors, such as dose-limiting side effects, lack of selectivity, tissue toxicity, limited drug access to tumor tissues, reduction of effective drug doses, and the emergence of multiple drug resistance with conventional or combination chemotherapy ${ }^{[27]}$. 


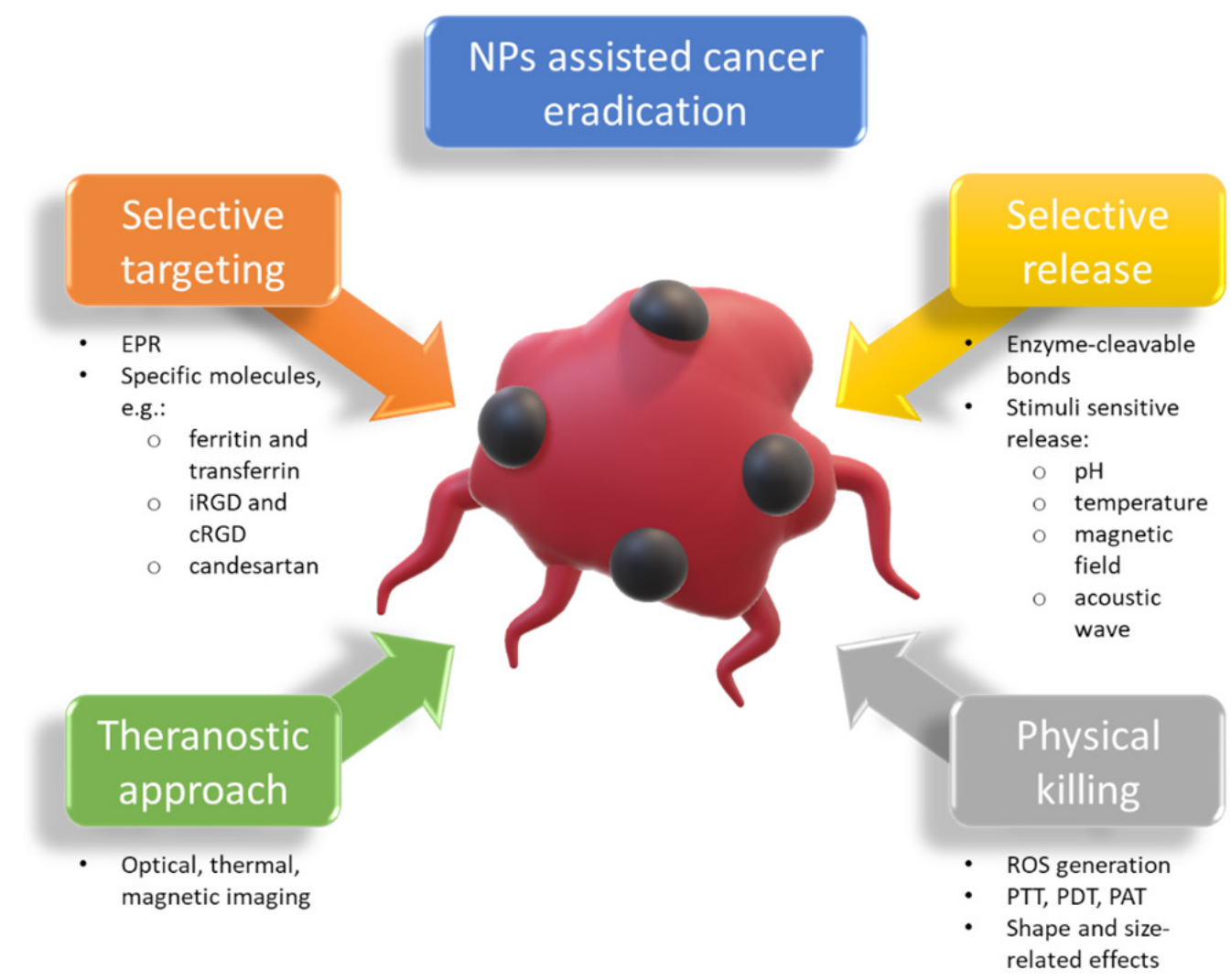

Figure 2. A graphical representation of NP-assisted cancer-killing effects. NP: nanoparticle; EPR: enhanced permeability

Nanoparticles (NPs) can be designed in a wide range of sizes, shapes, and compositions to be used for passive or active targeting of cancerous cells. Throughout the years, different strategies of drug delivery have been elucidated, including active targeting where NPs directly interact with cells or bind with specific receptors or antibodies expressed on the cells. Alternatively, the use of passive targeting is based on the enhanced permeability (EPR) effect. The EPR effect is the ability of molecules of 100-1000 nm in size to accumulate in the leaky vasculature exhibited on tumor tissue ${ }^{[27]}$.

Once the loaded NPs accumulate in the desired target, they can release the drug through different stimuli, such as temperature or $\mathrm{pH}$ change, respond to enzymes or antigens, or physical stimuli. Physical stimuli can include a magnetic field, light, or heat. This approach is gathering considerable research interest due to its higher versatility ${ }^{[28]}$.

Over time, more and more nanostructures have been discovered and designed, and their applicability in the field of nanomedicine has been extensively studied. Currently, different nanomaterials and nanoformulations, such as polymeric lipid and metal NPs, as well as nanovesicles (e.g., dendrimers and liposomes), have emerged as innovative, effective, and promising platforms for the treatment of drugresistant cancer cells ${ }^{[2]}$. Multiple benefits and mechanisms of NP-specific cancer-killing effects have been identified, and these are presented in Figure 2.

A few NP-drug formulations are already FDA approved, e.g., Abraxane (albumin NPs + PTX), GenexolPM (polymeric micelles + PTX), and Doxil (liposome + DOX). These NPs formulations provide better results than conventional drug treatments ${ }^{[30]}$. Nanocarriers have been reported to improve the therapeutic drug index, introduce the ability for multifunctional treatments, divert the ABC-transporter mediated drug 


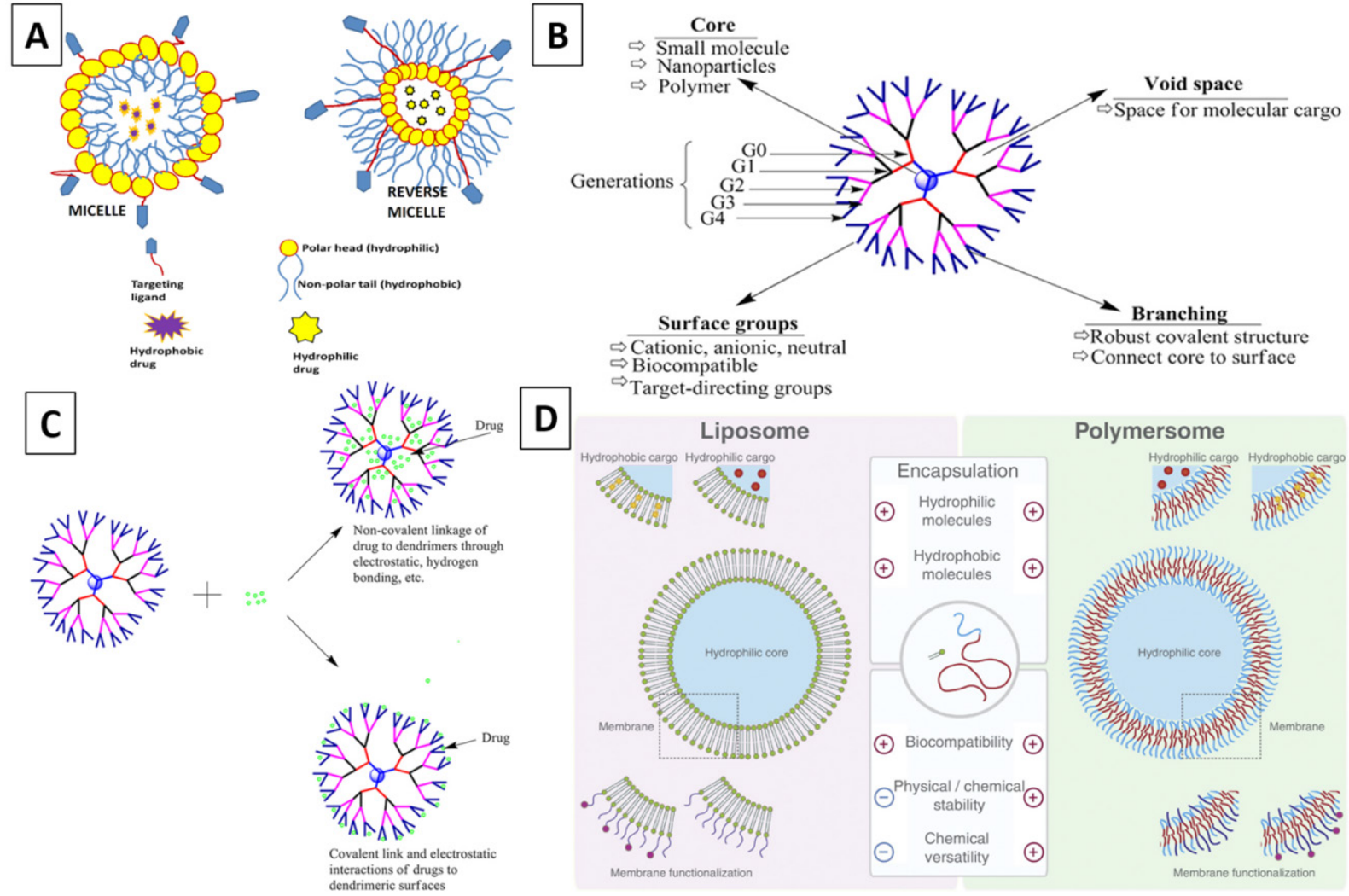

Figure 3. Schematic representation of polymeric micelle and reverse polymeric micelle (Original figure) (A); Schematic representation of a dendrimer $(B)^{[38]}$; Schematic representation of possible types of drug-dendrimer interactions $(C)^{[38]}$; Schematic representation of liposomes (left) versus polymersomes (right) $(D)^{[47]}$

efflux mechanism and selectively target the tumor cells, cancer stem cells, tumor-initiating cells, or cancer microenvironment ${ }^{[31]}$.

In the following sections, different nanocarriers will be explored for their effectiveness in fighting the progression of cancer-resistant tumors.

\section{Polymeric nanocarriers}

\section{Polymeric micelles}

Polymeric micelles (PM) are colloidal particles of a self-assembling nature. They have multiple interaction points compared to conventional micelles, and their structure comprises of two parts: the hydrophilic head and the hydrophobic tail ${ }^{[32]}$. Figure $3 \mathrm{~A}$ represents a schematic illustration of PM and reverse PM that can be utilized for drug delivery systems.

Tumor targeting with the help of micelles can be achieved by ligand-mediated targeting (folate, carbohydrate, proteins, peptides, and monoclonal antibodies), internal stimuli-responsive PM (pHresponsive $\mathrm{PM}$, enzyme-responsive $\mathrm{PM}$, and thermoresponsive $\mathrm{PM}$ ), and external stimuli-responsive $\mathrm{PM}$ (magnetic field-responsive PM, light-responsive PM, and ultrasound-responsive $\mathrm{PM})^{[33]}$.

Moreover, micelles can be made of many different polymers and biopolymers, all of which were proven effective in targeting metastatic or chemo-resistant cancers. For example, chitosan PM, a biopolymeric structure, was coupled with stearic acid-containing DOX and injected intravenously in breast tumorbearing BALB/c mice to study the uptake of the drug by the circulating monocytes. Almost $94 \%$ of 
the circulating monocytes took up the injected PM, which was visualized with the help of fluorescent molecules. Then, it was exocytosed by macrophages and taken up by $4 \mathrm{~T} 1$ cancer cells, which shows the efficacy of the treatment method ${ }^{[34]}$.

Alternatively, synthetic polymers, PEG, and its derivatives are most commonly used to form PM. Due to their high versatility, good biocompatibility, and ease of modification, PEG-based PMs have proven to be useful in the selective targeting of drug-resistant cancers. Yao et al. ${ }^{[35]}$ have developed PEG-based polymeric micelles, which were cleaved by matrix metalloproteinase-2 (MMP2), the enzyme involved in cancer progression and metastasis, for selective release of PM's payload (DOX and dasatinib) in cancer cells. The formulated micelles showed significant cytotoxicity against drug-resistant NCI/ADR-RES and MES-SA/ Dx5 cell lines. The authors confirmed that the formulated micelles could effectively inhibit the drug efflux caused by dasatinib and improve drug efficacy in MDR cells with DOX when used as a co-administered efflux inhibitor or as a micellar drug nanocarrier ${ }^{[35]}$.

Another example of PM-assisted targeting drug-resistant cancer can be found in a study by Gao et al. ${ }^{[26]}$ Herein, PEG-based PMs were used to promote ferroptosis in PCC cells. Two separate factors were used to induce ferroptosis: conjugation of arachidonic acid (ferroptosis precursor) and encapsulation of RSL3, glutathione peroxidase 4 (GPX4) inhibitor, arresting lipidic repair. The drug-carrier was postulated to accumulate in cells via the EPR effect and selectively release its cargo in reactive oxygen species (ROS)rich cancer environments. As a result, a 30-fold increase in human ovarian adenocarcinoma cells' killing efficiency was observed in vitro and as compared with control micelles. In vivo, selective accumulation in cancer and effective payload release resulted in improved survival rates, connected with tumor growth inhibition, and no adverse side effects were observed ${ }^{[26]}$.

An example of multifunctional PM can be found in the study by Zhen et al. ${ }^{[36]}$, in which PEG-based PMs [polymeric prodrug (PMP)] were encapsulated with novel NIR fluorophore (DEB-BDTO) as a photosensitizer along with a drug efflux pump inhibitor - TQR and PTX. As a result, an efficient PDT enabling drug carrier was fabricated, which selectively releases a chemotherapeutic drug and resistance development inhibitor upon NIR irradiation. The formulated micelles showed a prominent synergistic lethal effect when paired with photodynamic therapy and chemotherapy against resistant SKOV-3/MDR, in both in vitro and in vivo mice models. Tumor growth inhibition was observed for up to 17 days, and no adverse side-effects were identified ${ }^{[36]}$. In conclusion, the studies mentioned above suggest that careful tailoring of the PM compositions can be used to reverse the drug resistance and effectively target tumors.

\section{Dendrimers}

Dendrimers are nano-sized, 3D highly branched compounds ${ }^{[37]}$. They comprise three major parts: a central core, repeating units of continuously branched dendrons, and functional groups. Functional groups are located at the periphery of the dendrimer where modifications can be made, and the drug compounds can be attached to the dendrimer ${ }^{[38]}$. Figure $3 \mathrm{~B}$ represents a schematic illustration of a dendrimer that can be utilized for drug delivery systems, and Figure $3 \mathrm{C}$ illustrates possible types of drug-dendrimer interactions.

Dendrimers are generally employed for in vitro diagnostics, drug and vaccine delivery, gene transfection, and protein mimicking ${ }^{[39]}$. Common dendritic molecules used in the drug delivery applications are polyamidoamine (PAMAM), polypropylene imine (PPI), poly-L-lysine (PLL), polyglycerol, and polyglycerol-co-succinic acid. Of the dendrimers listed above, PAMAM and PLL are the most commonly used. The great advantage of using dendrimers lies in their good biocompatibility, combined with extremely high drug loading capacity ${ }^{[40]}$ and excellent delivery efficiency, which allows deep penetration of the drug into the tumor ${ }^{[41]}$. Stable systemic circulation and selective release of the drug at the tumor vasculature can be obtained by fabricating multi-component materials ${ }^{[42,43]}$. Also, multi-component materials can be combined with PDT therapy ${ }^{[44]}$. 
As for targeting drug-resistant cancers, dendrimers were found to provide excellent outcomes. Pan et al. ${ }^{[45]}$ formulated a PAMAM dendrimer with a PEG copolymer. When this dendrimer was co-loaded with DOX and therapeutic siRNA (siMDR-1), the formulated material showed significant anticancer efficacy against MDR human ovarian carcinoma (A2780/ADR) and breast cancer (MCF7/ADR). The authors confirmed the downregulation of P-glycoprotein in treated cells, which led to overcoming the resistance towards $\mathrm{DOX}^{[45]}$. Additionally, Gouveia et al. ${ }^{[46]}$ formulated polyalkylidenimine dendrimers functionalized with the organometallic moiety $\left[\mathrm{Ru}\left(\eta^{5}-\mathrm{C}_{5} \mathrm{H}_{5}\right)\left(\mathrm{PPh}_{3}\right)_{2}\right]^{+}$. The system showed significant anticancer efficacy against a cisplatin-resistant human ovarian carcinoma (A2780cisR) cell line.

\section{Polymersomes}

Polymersomes are a type of drug vehicle that have an aqueous interior and bilayer chambers that can carry both hydrophilic and hydrophobic drug molecules. These nanocarriers are synthetic analogs of liposomes and are made of amphiphilic block copolymer membranes [Figure 3] ${ }^{[47]}$. Polymersomes are usually copolymerized with PEG, and the formed copolymer can be linked to the hydrophilic, hydrophobic, or biological blocks for efficient drug delivery applications ${ }^{[48]}$. Usual block compositions are polybutadieneblock-ethylene oxide (PB-b-PEO) ${ }^{[49]}$, polycaprolactone (PEG-b-PCL) $)^{[50]}$, and folic acid-poly-L-glutamic acid-block-poly- $\varepsilon$-caprolactone $[\text { FA-PGA-b-PCL }]^{[51]}$.

Impressively, polymersomes exhibit greater versatility and stability than liposomes, with properties easily steered by the composition and molecular weight of co-polymers ${ }^{[47,52]}$. They are biodegradable so that they can help in the sustained release of the desired drug molecule in the biological environment ${ }^{[33]}$. Also, they have already been successfully employed in carrying certain anti-cancer drugs like PTX for enhanced intraperitoneal chemotherapy, GEM for lung cancer, and DTX for breast cancer ${ }^{[54-56]}$.

For instance, polymersomes were successfully used for combinational chemotherapy, where PTX and DOX were employed for treating hepatocellular carcinoma. The hydrophobic core of the polymersomes contained hydrophobic drugs: PTX and DOX, while the outer surface was formed by a dense, hydrophilic PEG-folate corona. Such composition allowed for good dispersibility and selective targeting of cancer cells through folate receptors. The particles were readily and selectively up-taken by BEL-7404 cells in vivo, followed by a sustained $\mathrm{pH}$-responsive release of the drug. As a result, tumor growth was slowed down by $80 \%$, with no adverse side effects observed ${ }^{[52]}$.

Qin et al ${ }^{[57]}$ formulated a folate-decorated a triblock copolymer $\mathrm{PCL}_{7500}-\mathrm{ss}-\mathrm{PEG}_{7500}-\mathrm{ss}^{\mathrm{S}-\mathrm{PCL}_{7500}}$ based redoxresponsive polymersome, which was loaded with TQR, DOX, and PTX. The formulated polymersome exhibited elevated drug accumulation into MCF-7/ADR cells via the TQR-induced P-glycoprotein efflux inhibition, and the cell cycle was blocked in the G2/M phase.

\section{Summary}

Polymeric nanocarriers are a wide group of materials whose qualities can be easily tailored for meeting specific needs. Specifically, the time of degradation and size can be highly fine-tuned, and this is particularly useful for ensuring EPR and controlled release. However, their intrinsic properties do not allow for easy employment of a theranostic approach and combining multiple killing mechanisms to effectively fight chemoresistant or metastatic cancers. These features can be introduced through further modifications, which can be troublesome and hard to design. In particular, it is hard to ensure multiple binding mechanisms for different additives, and competing reactions can, therefore, be expected. For these reasons, examples of multifunctional polymeric nanocarriers are rather scarce in the literature, mostly concerning encapsulation of more than one additive. Still, there are some examples of excellent studies that have undertaken the goal of combining multiple actions in elegant, carefully designed carriers, and these are studies by $\mathrm{GaO}^{[26]}$ and Zhen ${ }^{[36]}$. High cancer-killing efficiency, selectivity, and ability to completely reverse 
A

TMV delivery of cisplatin restores efficacy in OVCA

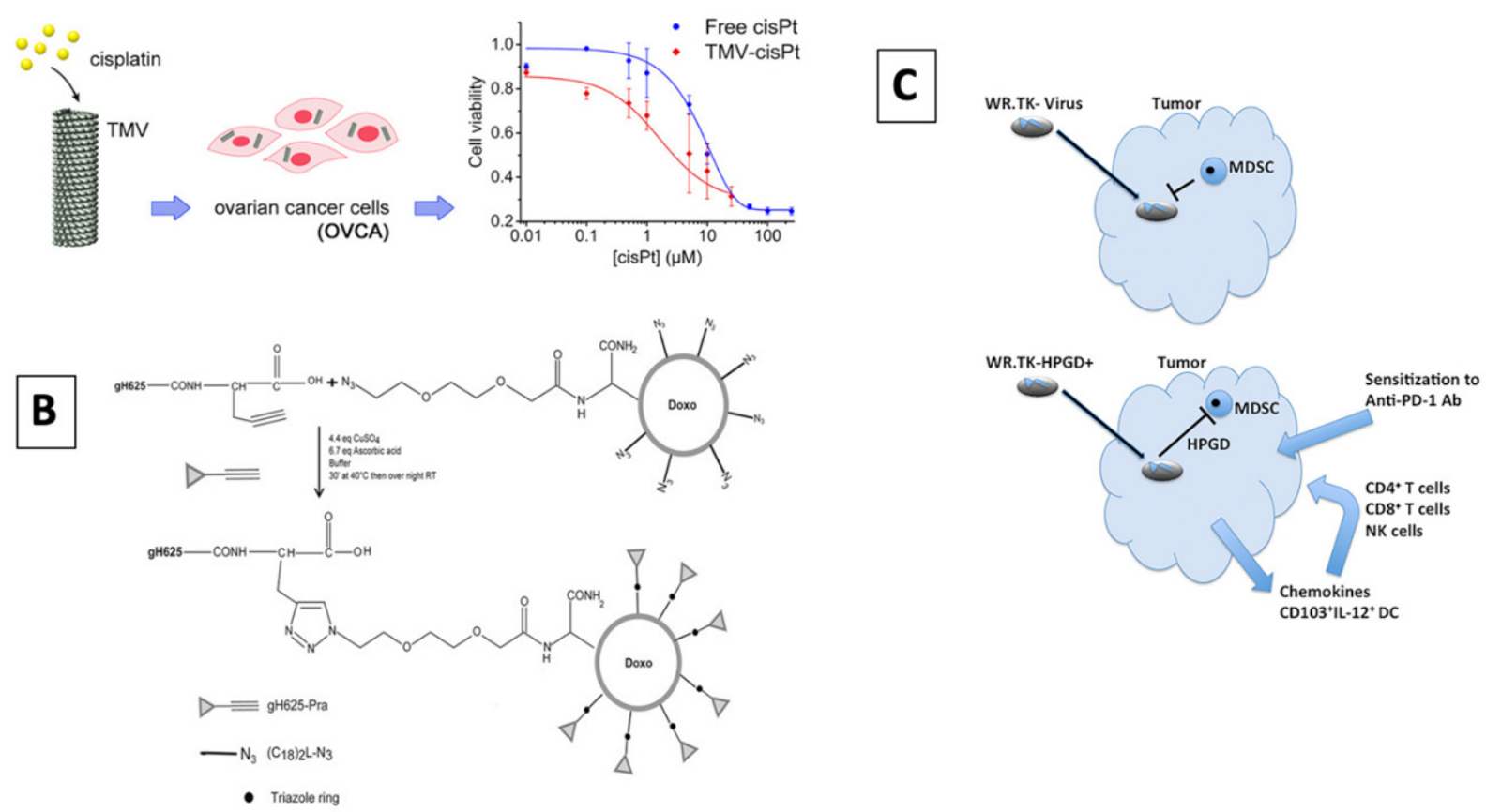

Figure 4. Tobacco mosaic virus (TMV) delivery of Pt in platinum-resistant ovarian cancer cells (A) ${ }^{[58]}$; DOX delivery in gH625 Herpes virus derived-protein encapsulated liposome in drug-resistant lung adenocarcinoma cells $(B)^{[59]}$; Western reverse (WR) vaccinia targeting PGE2 to overcome immunotherapy resistant cancer cells (C) ${ }^{[61]}$

the chemoresistance prove that such an approach should be treated as guidance for further development of polymeric nanoparticles. In the future, more strategies that would allow the introduction of a theranostic approach should also be pursued.

\section{Viral nanocarriers}

Viral nanocarriers, also known as viral-based nanoparticles (VNPs) or virus-like particles (VLPs), are virus-based materials that present a nanometric size with a specific geometry [Figure 4A]. They are called viral nanocarriers as they are constructed with viruses or viral proteins acting as nanocages; hence, their potential to be used as drug delivery carriers, imaging, gene therapy, or vaccination purposes. VNPs are highly versatile, allowing multiple morphological and surface chemistry modifications while being highly biocompatible ${ }^{[31]}$.

VNPs have demonstrated their potential to overcome possible resistance to chemotherapy drugs. For instance, VNPs made by tobacco mosaic virus (TMV) loaded with Pt showed enhanced efficacy towards both platinum-resistant and platinum-sensitive ovarian cancer cells. The encapsulation of the drug within the viral structure allowed for increased efficacy and overall lower dosage with superior cytotoxicity and DNA-double strand breakage in vitro ${ }^{[58]}$. The use of virus-derived proteins has also shown efficacy against chemotherapy-resistant tumors. The reason behind this is related to the ability of these proteins to cross the cellular membrane, conferring a directed drug-delivery character to the therapy. As an example, a gH625 protein, derived from Herpes simplex virus 1 (HSV-1), was conjugated to DOX (DOX) and encapsulated into a liposome [Figure 4B]. The as-received system was able to induce apoptosis in the DOX-resistant lung adenocarcinoma cell line. The authors demonstrated that it was the presence of the viral particle, which allowed the enhanced delivery to overcome the drug resistance ${ }^{[59]}$. 
Moreover, oncolytic viruses (OV), VNPs that are based on living viruses, can provide unique cancerfighting strategies that are characterized as immunotherapy since they can replicate inside the tumor, allowing them to spread along with the tissue. OV proliferate inside the tumor using multiple pathways, inducing immunogenic cell death without causing any effect on healthy tissue ${ }^{[60]}$. For that reason, OV is a rising interest in the cancer drug resistance field. The use of oncolytic vaccinia has been studied in recent years. Patients generally present highly variable immune defects and suppression, which lead to increased cancer resistance. Recent investigations show that molecules such as prostaglandin E2 (PGE2) play vital roles in cancer cell resistance.

Some viral vectors can target PGE2 and reverse localized immunosuppression. In their research, Hou et al. ${ }^{[61]}$ used a modified-Western Reverse strain of vaccinia virus against multiple immunotherapy-resistant cancers in mice. The viral formulation expressed hydroxyprostaglandin (HPGD), which successfully targeted PGE2 and depleted granulocytic myeloid-derived suppressor cells (G-MDSC) to overcome resistance. Other studies indicated that the endoplasmic reticulum (ER) stress response could reprogram resistance in some cancer cell lines using oncolytic viruses like Maraba rhabdovirus. The main aim of the study was to sensitize cancer cells to OV and make the therapy more efficient and effective using a small molecule inhibitor called IRE1 $\alpha$. Glioblastoma tumors undergo an enhanced caspase-derived apoptotic cell death when in contact with the viral agent, as tested in murine models ${ }^{[62]}$.

Vesicular stomatitis virus (VSV) is par excellence in the prototypic OV. Nonetheless, some studies have shown an increased cancer cell resistance to it. Ongoing investigations suggest that the use of some inhibitors can be successfully applied to reverse this effect in prostate, ovarian, or glioblastoma cancer. Upon silencing histone deacetylase (SIRT1), prostate cancer cells become sensitive to the virus and allow its replication and spread $^{[63]}$.

Similarly, the use of interferon modulators in a VSV modified with a glycoprotein from lymphocytic choriomeningitis virus (GP) allowed the VSV to overcome partial resistance in ovarian cancer cells. The combination of GP-VSV with the inhibitor ruxolitinib showed enhanced cytotoxicity towards ovarian cancer cell lines without damage to healthy tissues or signs of resistance or remission ${ }^{[64]}$. Similar types of modulators derived from different kinds of viruses, e.g., the Semliki Forest virus, were previously applied successfully in glioblastoma ${ }^{[65]}$.

\section{Summary}

The use of VNPs or OVs to fight chemoresistant cancers is a relatively new approach that has not yet been well studied. Still, it seems to be very promising, and multiple studies report efficient eradication of cancer cells, while the main advantage lies in VNPs' ability to easily and selectively penetrate the cells. Some of the cited studies report excellent efficiency in cancer eradication in vivo upon intratumoral ${ }^{[61,64]}$ or intraperitoneal ${ }^{[6,65]}$ injections in tumor-bearing mice models. The treatment was able to either arrest the tumor growth ${ }^{[61,62,64]}$ or eradicate it ${ }^{[65]}$. What is more, VNPs or OVs can provide long-term immunity against tumor regrowth. Nonetheless, there is still a lot of investigation to cover to apply these techniques, such as: understanding the tumor heterogeneities, analyzing the in vivo toxicities, and analyzing long-term effects. Specifically, in the cited studies, severe side effects in animal models were reported, with viral escape and transfection of healthy tissues observed, followed by neurological symptoms. Such reactions should be properly identified, and mitigating steps need to be designed before the carriers can be regarded as safe and be qualified for further studies. One must also take into account that VNPs or OVs alone will not allow for employing combinatorial approaches and instead should be treated as an addition to other strategies. For example, they could be loaded inside a certain carrier along with other compounds, thus permitting theranostic and/or multiple cancer cell killing mechanisms. 


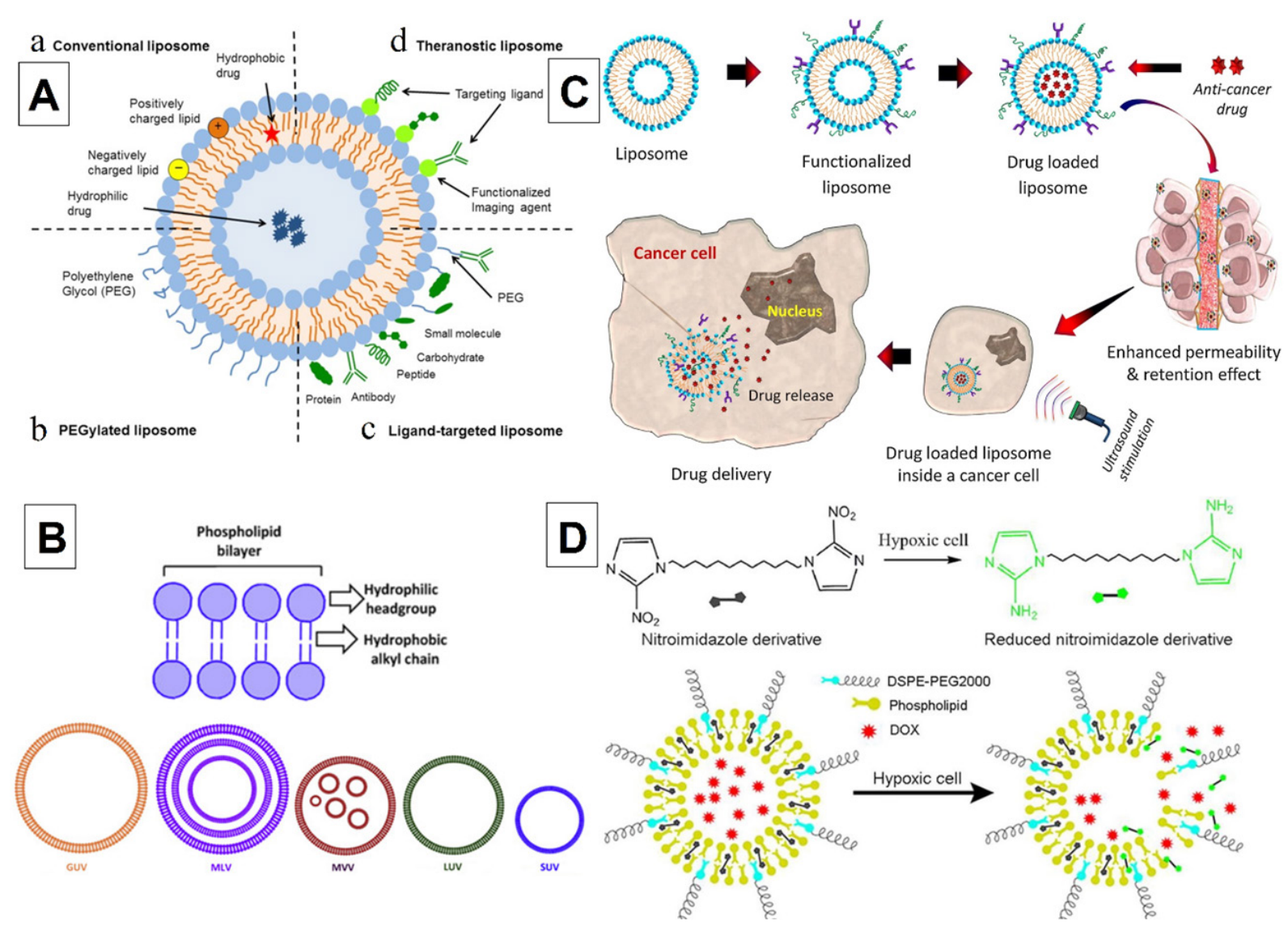

Figure 5. Schematic representation of the different types of liposomal drug delivery systems. Conventional liposome (a), PEGylated liposome (b), Ligand-targeted liposome (c), Theragnostic liposome $(d)^{[72]}(A)$; Schematic representation of the different types of liposomes $^{[67]}$ (B); Schematic representation of liposome-based drug delivery system for cancer therapy ${ }^{[68]}$ (C); Schematic illustration for triggered release of hypoxia-responsive liposomal drug delivery system $(D)^{[196]}$

\section{Lipid nanocarriers}

\section{Liposomes}

Liposomes are spherical phospholipid vesicles consisting of one or more phospholipid bilayers enclosing an aqueous core and are biomimetic to natural biological membranes ${ }^{[6-69]}$. Liposomes are biodegradable, biocompatible, nonimmunogenic, and possess a high loading capacity. With their amphiphilic nature, liposomes can deliver both hydrophilic (inside the core) and hydrophobic (embedded inside the phospholipid bilayer) drugs ${ }^{[69-71]}$. However, these particles have some shortcomings: instability, short blood circulation time, and insufficient drug loading capacity. To overcome these issues, PEGylated liposomes were developed, and these can be further modified with different therapeutic and diagnostic agents as well as antibodies, proteins, carbohydrates, vitamins, and glycoproteins for targeted drug delivery systems $[\text { Figure } 5 \mathrm{~A}]^{[72]}$.

Due to their excellent biocompatibility, liposomes were amongst the first studied cancer drug carriers, with some formulations already FDA-approved: Doxil/Caelyx ${ }^{\circ}$ (pegylated liposomal DOX in the range of $100 \mathrm{~nm}$ ), Marqibo $^{\circ}$ (vincristine sulfate liposome injection in the range of $100 \mathrm{~nm}$ ), Onivyde ${ }^{\bullet}$ (IRI liposome injection in the range of $110 \mathrm{~nm}$ ), Vyxeos ${ }^{\bullet}$ (liposomal cytarabine/daunorubicin in the range of $107 \mathrm{~nm}$ ), and DaunoXome $^{\oplus}$ (daunorubicin citrate liposome injection in the range of 40 to $\left.80 \mathrm{~nm}\right)^{[73]}$.

Their usage is already well established, and recent efforts are focused on enhancing their selectivity, which is typically completed by using $\mathrm{pH}$-sensitive systems, modifying the materials with cancer-specific molecules, 
or introducing additional molecules that can combat the chemoresistance. Some studies combine all three approaches. One example is a study by Paliwal et al. ${ }^{[74]}$, who reported that DOX-loaded hyaluronic acidtargeted $\mathrm{pH}$-sensitive liposomes were more efficacious than free DOX after $48 \mathrm{~h}$ of treatment against CD44 receptor over-expressing MCF-7 cells with the $\mathrm{IC}_{50}$ values of 1.9 and $3.2 \mu \mathrm{mol} / \mathrm{L}$, respectively.

Additionally, the in vitro release investigation revealed the $\mathrm{pH}$-dependent release of DOX from the formulated drug-loaded liposomes, with faster release at mildly acidic $\mathrm{pH} \sim 5$ in comparison to physiological $\mathrm{pH} \sim 7.4$. In vivo performance was tested in skin tumor-bearing mice. The carrier was found to reduce the DOX accumulation in vital organs, revealing reduced systemic toxicity. At the same time, effective targeting of tumors resulted in its size reduction by $80 \%$ on the 30 th day of experimentation ${ }^{[74]}$.

In a different study, Chen et al. ${ }^{[75]}$ formulated a $\mathrm{pH}$-sensitive liposomal system in which positively charged liposomes (for easier cellular internalization) were covered with a negatively charged and $\mathrm{pH}$-sensitive polymer (PEG-PLL-DMA), which was responsible for improving the drug carrier blood circulation time. The formulated materials showed a charge-reversal effect: they were negatively charged under a physiological $\mathrm{pH}$ value of 7.4 , but the charge changed to positive in a $\mathrm{pH}$ value of 6.5 (in tumorous tissues). This was due to the cleavable amide linkages formed between the PEG-PLL and DMA. Positively charged carriers could then be easily internalized by cancer cells where the carriers could release cargo, specifically, NO donor diethylenetriamine diazeniumdiolate (DETA NONOate), for inhibiting the efflux pump and PTX, for cytotoxic effect against cancer cells. The in vivo studies on A549/T tumor-bearing mice (PTX resistant cells) revealed that the obtained system improved the drug's accumulation and residency time inside the tumor. This leads to significant tumor necrosis and size reduction compared to a drug administered without a carrier. Additionally, it was observed that side effects towards vital organs were $\operatorname{mild}^{[75]}$.

Another strategy to fight drug-resistant cancer is to combine multiple drugs into one liposomal carrier. The most common method involves combining a traditional chemotherapeutic drug with another molecule, whose role is to reduce or mitigate resistance mechanisms. Among different compounds, two anti-malaria drugs, dihydroartemisinin (DHA) and chloroquine phosphate (CP), have been reported to participate in the process of cell sensitizing. The former has been suggested to inhibit the mTOR (mammalian target of rapamycin), responsible for pro-survival mechanisms in cells ${ }^{[76]}$, while the latter inhibits the efflux transporters $^{[77]}$.

The therapeutic effect can then be further boosted by using cancer-selective molecules. In a study by Kang et al. ${ }^{[78]}$, a mannosylated liposomal tumor-targeting co-delivery system showed significant anticancer activity by combining DHA with DOX against drug-resistant human colon cancer HCT8/ADR cells overexpressing the mannose receptor. The $\mathrm{IC}_{50}$ value was $0.073 \mu \mathrm{g} / \mathrm{mL}$, while free DOX or DHA did not show any cytotoxicity to this drug-resistant cancer cell line. In vivo studies in tumor-bearing mice revealed selective accumulation of the drug carrier in the tumor, leading to a nearly $90 \%$ tumor inhibition rate, as compared to $70 \%$ reported in combo drugs only. Furthermore, usage of the carrier was able to reduce the DOX-related side effects in the form of hepatic damage.

Meanwhile, Qiu et al. ${ }^{[77]}$ reported effective anticancer activity of a composite liposomal system coencapsulating PTX with CP to treat PTX-resistant carcinoma. The authors confirmed that their synthesized liposomal system could block the efflux of PTX by ABC transporters. The authors proved that using multiple drugs in one system is far more effective than administering them in separate carriers. This was tested in an in vivo mice model, where tumor size inhibition was two times more significant than in the case of single or separately administered drugs, which was explained by a synchronized internalization pattern. 
Other compounds that can be used to reduce the drug pumping in the liposome-based carriers out are coumarin, TGPS, or hydroxypropyl- $\beta$-cyclodextrin $(\mathrm{HP}-\beta-\mathrm{CD})$. In a study by Li et al. ${ }^{[79]}$, liposomes were co-loaded with coumarin and DOX and coated with TGPS, and their efficiency was tested against resistant A549/DDP cells, both in vitro and in vivo, as a xenograft tumor in the mice model. The materials were found to be readily up-taken by cancer cells, reducing their viability by up to $90 \%$. The $\mathrm{IC}_{50}$ was nearly two times lower than in the commercially available drug, Taxotere ${ }^{\bullet}$. In vivo, the tumor growth was almost completely arrested, with no adverse side effects observed. By comparing the system to the FDA-approved drug, the scientists were able to prove the excellence of their material.

Similarly, promising results were reported by Shen et al. ${ }^{[80]}$. In this study, liposomes were co-loaded with HP- $\beta$-CD and administered to fight PTX-resistant A549/T lung cancer cells, both in vitro and in vivo (mice model). With the $\mathrm{IC}_{50}$ reduced 2-fold as compared to free PTX and significantly enhanced apoptosis induction, the system was able to completely inhibit the tumor growth, with no adverse side effects observed.

While most drug carrier strategies are focused on improving the circulation time and cancer cell internalization while inhibiting the drug efflux mechanisms, some are even more fine-tuned. This approach can be found in a study by Li et al. ${ }^{[81]}$, in which the authors had decided to focus on improving the nuclear uptake of DOX. To do so, an AS1411 aptamer with high nucleolin binding affinity (overexpressed in the cancer cell nucleus) was complexed with DOX and loaded into the liposomes. The obtained materials were evaluated for their efficiency in eradicating drug-resistant breast cancer (MCF-7/ADR) cells in vitro. The authors confirmed that DOX-Ap liposomes bound with nucleolin strongly and eventually accumulated in the nuclei to effectively kill the MCF-7/ADR cancer cells. Compared to pure DOX, the $\mathrm{IC}_{50}$ value was reduced nearly 5 -fold.

\section{Solid lipid nanoparticles}

Solid lipid nanoparticles (SLNs) are an emerging generation of colloidal nanocarriers that consist of surfactant-stabilized biodegradable lipids based on a solid lipid matrix ranging from 50 to $1000 \mathrm{~nm}^{[82]}$. As shown in Figure 6A, the SLNs comprise the solid lipid matrix, surfactants, and occasionally cosurfactants $^{[83]}$. Typically, waxes (e.g., cetyl palmitate), triglycerides (e.g., tripalmitin), steroids (e.g., cholesterol), fatty acids (e.g., decanoic acid), and partial glycerides (e.g., glyceryl behenate) are the most used lipids ${ }^{[84]}$. A drug-enriched shell, drug-enriched core, and solid solution (homogeneous matrix) are three possible models of SLNs-based drug carriers, depending on differences in the melting point between drugs and lipid matrices [Figure $6 \mathrm{~B}]^{[85]}$.

The advantages of SLNs for drug delivery systems include the utilization of biocompatible and biodegradable ingredients, the lack of application of organic solvents, drug protection from environmental damage, high physical stability, ease of preparation, controlled drug release, and ability of CNS targeting ${ }^{[86]}$. SLNs have been successfully used to encapsulate lipophilic and hydrophilic medicines ${ }^{[87]}$, peptides/ proteins ${ }^{[88]}$, and nucleic acids ${ }^{[89]}$. In recent years, the use of SLNs for anticancer drug delivery has become a rapidly growing field of study ${ }^{[90,91]}$.

When applying SLNs to fight drug-resistant cancers, usually a combination of more than one drug, the addition of a surfactant to inhibit the drug efflux pumping out or usage of specific cancer cell-targeting molecules was used. More advanced studies suggest a combination of either two or all three approaches. Affram et al. ${ }^{[92]}$ suggested a GEM-loaded SLN, fabricated from glyceryl monostearate (GMS), using two different surfactants in varying ratios: polysorbate 80 (Tween ${ }^{\circ} 80$ ) and poloxamer 188 (Pol 188). Herein, the surfactants were used to inhibit the drug efflux mechanisms. Among the twenty-four formulations tested in the study, the best one was found to be $1.0(\mathrm{w} / \mathrm{v} \%)$ GMS, 3.5 (w/v\%) Tween 80, and 0.05 (w/v\%) GEM with 


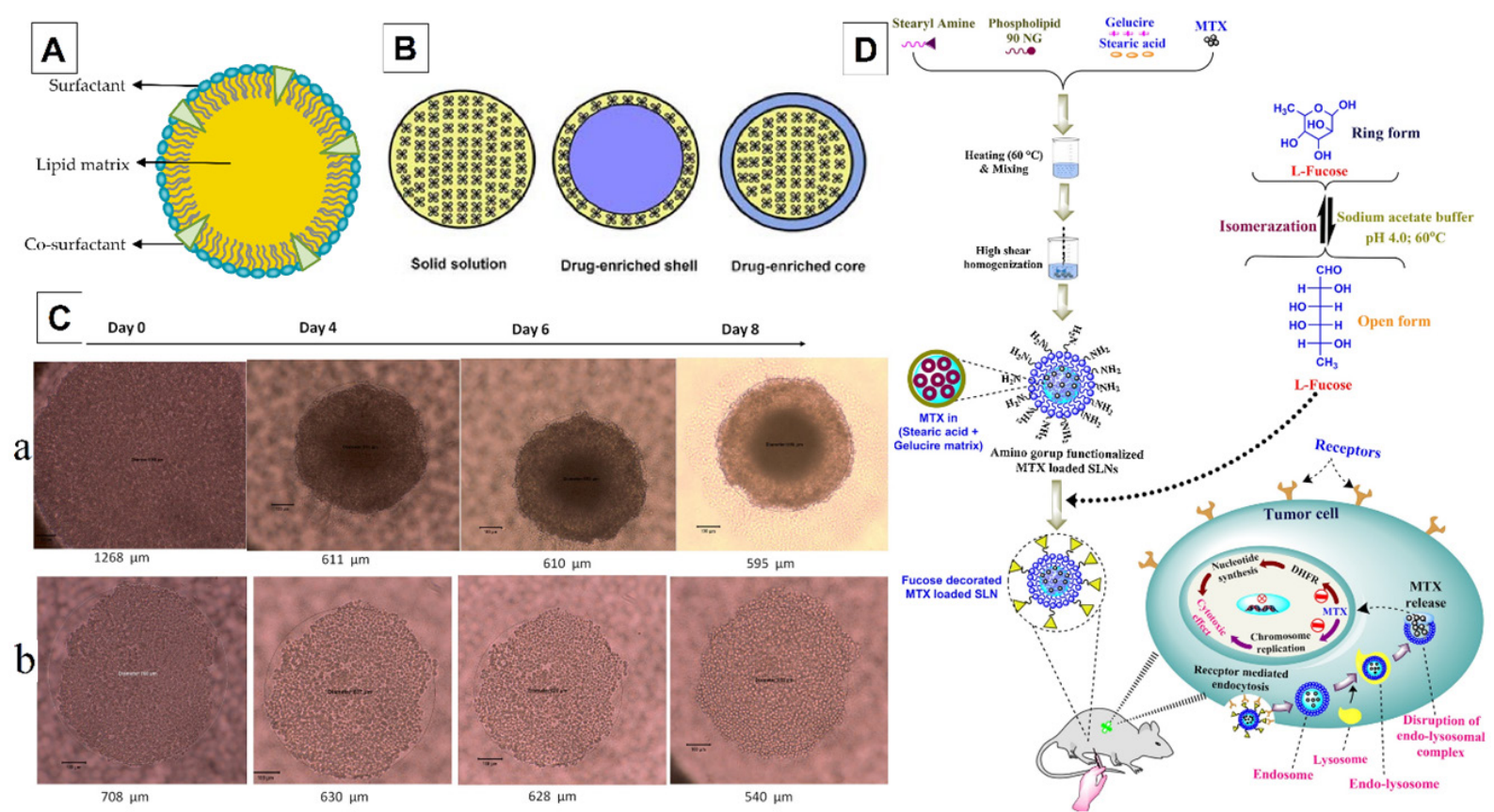

Figure 6. Schematic representation of the SLNs structure, representing the solid lipid matrix, surfactant, and co-surfactant ${ }^{[83]}$ (A); Classification of three types of classical SLNs including drug-enriched shell, drug-enriched core, and solid solution ${ }^{[85]}$ (B); Images of spheroids formation in different period of time with respective size $(\mu \mathrm{m})$. MCF-7/ADR (a), NCI/ADR (b). Images are representative of triplicate samples ${ }^{[93]}$ (C); Development of fucose decorated methotrexate loaded SLNs (D). The proposed mechanism of action is methotrexate via ligand-receptor mediated endocytosis and internalization ${ }^{[96]}$

an average particle size of $603 \pm 19 \mathrm{~nm}$ and entrapment efficacy of $68.3 \% \pm 4.8 \%$. This material exhibited higher cytotoxicity against the patient-derived primary pancreatic cancer cell line (PPCL-46) and human pancreatic Mia-PaCa-2 cells, compared to free GEM using monolayer (2D) and spheroid (3D) cell cultures. In $2 \mathrm{D}$ cultures, the $\mathrm{IC}_{50}$ values were reduced 3-fold for Mia-PaCa-2 and over 4.5-fold for the in PPCL-46, compared to pure GEM. In 3D cultures, these values were two times and four times lower, respectively.

In a more complex study, Oliveira et al ${ }^{\left[{ }^{93]}\right.}$ formulated SLNs, co-loaded with DOX, and $\alpha$-Tocopherol succinate (TS) (DOX-TS-SLNs), with sizes in the range of 74 to $80 \mathrm{~nm}$ and entrapment efficacy of $99 \%$. The aim was to obtain the synergistic effect, determine the ability to kill chemoresistant cells by disrupting their nuclei and mitochondria, and induce apoptosis. To improve internalization and reduce the drug efflux, materials were also coated with tocopheryl polyethylene glycol succinate (TPGS) as a surfactant. Efficiency was tested against drug-resistant cancer cells (breast MCF-7/ADR and ovarian NCI/ADR) growing in monolayer and spheroid cultures. The materials revealed significant cytotoxicity towards MCF7/ADR and NCI/ADR cells. The cytotoxicity was higher when a combo drug was administered than when a single-drug loaded carrier was applied. In $2 \mathrm{D}$ cultures, the $\mathrm{IC}_{50}$ values were found to be 3 -4-fold reduced for combo drugs compared to DOX. In 3D cell cultures, nanoparticles easily penetrated the spheroids, reaching their center. A combination of two drugs was 1.5 times more effective in killing the NCI/ADR and 3.5 times more effective in killing the MCF-7/ADR when compared to DOX only. While further in vivo studies are necessary, the authors were able to suggest the good performance of the drug by demonstrating the efficiency of the system in $3 \mathrm{D}$ cultures.

Similarly, a pH-responsive hybrid drug delivery system was recently developed by conjugating $\mathrm{d}-\alpha$ tocopheryl polyethylene glycol 1000 succinate (TPGS), a kind of P-gp inhibitor, on the surface of laponite nanodisks to effectively treat DOX-resistant breast cancer cells (MCF-7/ADR) by inhibiting the activity of P-gp-mediated drug efflux and effectively accumulating DOX within cancer cells. In vivo results revealed that the formulation outstandingly suppressed MCF-7/ADR tumors with low side effects ${ }^{[94]}$. 
A similar system was suggested by Tang et al. ${ }^{[95]}$. This team co-loaded curcumin (Cur) and piperine (Pip) into SLNs covered with TPGS and Brij 78 molecules. While Cur is known to possess anticancer properties by downregulating survival pathways, its second mechanism of action involves reducing the drug efflux mechanisms. The latter is also a known mechanism of action of Pip, TGPS, and Brij. Thus, a safe system not involving the usage of chemotherapeutics was suggested for the effective treatment of MDR cancer cells. The system's performance was tested against the paclitaxel-resistant A2780/Taxol cell line. The studies revealed that Pip, TPGS, and Brij 78 are all able to enhance the therapeutic effect of Cur, which is the strongest for all the molecules combined - reaching over $60 \%$ inhibition in vitro. While these results seem promising, at their current stage, it is hard to evaluate their actual performance since the biocompatibility with healthy cells was not evaluated, and the ability to selectively accumulate in cancerous tissues was not studied.

Examples of studies that had employed cancer-cell specific targeting as an effective tool to fight chemoresistant cancer using SLN can be found in articles by Garg et al ${ }^{[96]}$, Wang et al ${ }^{[97]}$, and Zheng et al. ${ }^{[98]}$ The specific ligand molecules were fucose targeting lectin receptors, hyaluronic acid (HA) targeting CD44 and CD168 (RHAMM) receptors (both lectin and RHAMM receptors are overexpressed in the cellular wall of some cancers), and RGD sequence aimed at integrin $\alpha_{v} \beta_{3}$, which is overexpressed in vessels of various tumors types, respectively.

In the system proposed by Garg et al. ${ }^{[96]}$, SNL with a solid core of Gelucire ${ }^{\bullet}$ 50/13 and stearic acid and a solid lipid coating of phospholipid 90 NG and stearyl amine embedded with methotrexate (MTX) were fabricated and coated with fucose (FU) [Figure 6D]. The particles' average diameter was $174.51 \pm 5.1 \mathrm{~nm}$, with an entrapment efficiency of $84.3 \% \pm 1.24 \%$. In vitro performance was evaluated against MCF-7 cells, while, in vivo, a mice model was used with a pharmaceutically induced skin cancer. The FU coated materials had an improved cytotoxic effect against cancer cells, with the $\mathrm{IC}_{50}$ values reduced 5 -fold when compared to MTX and 2-fold when compared to uncoated particles, due to increased cellular uptake. In vivo, the drug was found to selectively accumulate inside the tumor, inhibiting its growth and granting a $100 \%$ survival rate up to 75 days post-treatment.

Moreover, in their study, Wang et al. ${ }^{[97]}$ combined materials that specifically targeted HA with pluronic 85 coatings for inhibiting drug efflux. These were tested against PTX-resistant cancers HeLa/PTX cervical and MCF-7/PTX breast cells in vitro. The materials easily accumulated inside the cancer cells, reducing their viability by up to $90 \%$ (for the highest concentration used). Compared to pure PTX, the $\mathrm{IC}_{50}$ for HeLa/PTX was reduced over 40 times. In the in vivo model, using HeLa/PTX tumor-bearing mice, the drug was found to be selectively accumulated in the tumor, arresting its growth, indicating that the suggested system might contribute a safe tool able to fight drug-resistant cancers.

Meanwhile, in a study by Zheng et al ${ }^{[98]}$, glycerin monostearate (GMS) was modified with a pH-sensitive liner [adipic acid dihydrazide (HZ)], which was linked to RGD and used to encapsulate DOX. Thus, cancer cell targeting drug carriers that release the drug under the slightly acidic $\mathrm{pH}$ of cancerous tissues was made. It was found that the proposed modification enhances the cellular uptake of drugs in MCF-7 and MCF7/ADR cells, leading to a significant reduction of the $\mathrm{IC}_{50}$ values in contrast with pure DOX, an 8 -fold reduction in MCF-7 and an 11 -fold reduction in MCF-7/ADR cells was observed. Interestingly, the $\mathrm{IC}_{50}$ value of the carrier was similar regardless of cell type, indicating that it is very efficient in overcoming drug resistance. In mice bearing MCF-7/ADR xenograft tumors, tumor growth was significantly arrested when compared to pure DOX treatment, which was ineffective. No changes in body weight and non-significant accumulation of the drug carrier throughout the vital organs indicates that the carrier improves the efficacy while reducing the negative side-effects of a DOX-based treatment. 


\section{Summary}

In short, various lipid nanocarriers have a high potential to become the new, standard strategy to treat chemoresistant cancer cells. The specific characteristic of these nanocarriers includes biocompatibility and selectivity. In particular, liposomes can increase the therapeutic efficacy of anti-cancer agents, while SLN in combination with surfactants, contributes to overcoming the drug efflux. Both types of lipid nanocarriers can be fairly easily modified with additional functionalities through ligand attachment, PEGylation, or the introduction of stimuli sensitive components. All of these features contribute to the fact that the lipid nanocarriers are currently one of the few nanocarriers cleared for clinical use (e.g., Abraxane, Taxotere). Still, as for polymeric or viral nanocarriers, the employment of theranostic approaches is elusive and requires multistep modifications, which can reduce the material's biocompatibility or treatment efficiency.

\section{Metal-based nanocarriers}

\section{Gold nanoparticles}

Undoubtedly, gold nanoparticles (AuNPs) are among the most widely employed nanosystems for these applications due to their high surface-to-volume ratio, hydrophilicity, and functionality, combined with high in vivo stability and a large surface area available for the attachment of materials such as antibodies.

Recently, Pedrosa et al. ${ }^{[99]}$ developed multifunctional AuNPs employed as vehicles for the delivery of the $\mathrm{Zn}(\mathrm{II})$-based coordination compound $[\mathrm{Zn}(\mathrm{DION}) 2] \mathrm{Cl} 2(\mathrm{ZnD})$ to increase the toxicity of the drug towards DOX-resistant colorectal carcinoma. Selective targeting was achieved through cetuximab which interacts with the epidermal growth factor receptor, typically overexpressed in cancer cells. The authors reported that the nanostructure could significantly inhibit cell proliferation and trigger the death of resistant tumor cells in vivo.

Similarly, Rathinaraj et al. ${ }^{[100]}$ developed a folate-gold-bilirubin (FGB) nanoconjugate used to efficiently reverse multidrug-resistance in P-expressing KB-ChR-8-5 cells. The FGB nanoconjugate was reported to elicit a stronger inhibition of the cells when compared to a treatment based on either bilirubin or AuNPs alone. The nanoconjugate was able to induce tumor suppression, ROS overexpression, DNA strand breaks, and apoptotic morphological changes in the cells, as tested in a mouse model.

Trastuzumab (Tmab), a monoclonal antibody that targets the human epidermal growth factor receptor 2 (HER2), is another good example of a component that has seen an increased resistance over time, as no current HER2-targeted therapeutic agent is effective against Tmab-resistant gastric cancer. Recently, HER2-targeted AuNPs were produced by successfully conjugating Tmab onto the surface of the NPs. After synthesis, the therapeutic effect of the AuNPs was tested towards HER2-positive Tmab-resistant (MKN7) or Tmab-sensitive (NCI-N87) gastric cancer cell lines, both in vitro and in vivo via intratumoral injection of the NPs. The anticancer effect was achieved via autophagy of the subcutaneous tumors ${ }^{[101]}$.

Deng et al. ${ }^{[102]}$ developed multifunctional AuNPs to simultaneously co-deliver three anticancer agents: AS1411, DOX, and anti-221, which are commonly used to enhance leukemia treatment efficacy. The NPs significantly inhibited the proliferation of the cells by inducing apoptosis, in a process associated with marked downregulation of the short RNA molecule miR-221 and the enzyme DNMT1. Besides, primary blasts obtained from the leukemia patients experiencing chemoresistant relapse were exposed to these NPs, becoming sensitized to DOX.

Pancreatic ductal adenocarcinoma (PDAC) has become one of the deadliest solid cancers found in patients worldwide. A recent report showed that $20 \mathrm{~nm}$ diameter AuNPs could sensitize pancreatic cancer cells to the anticancer drug gemcitabine through inhibiting migration and colony-forming ability of pancreatic cancer cells ${ }^{[103]}$. 
Similarly, it is well-known that HSP90 (a chaperone protein that assists other proteins to fold properly) inhibitors have the potential to treat many types of cancer due to the dependence of tumor cells on HSP9o for cell growth. Researchers found that Cullin-5 (Cul5) E3 ubiquitin ligase is needed for HSP90 inhibitors to induce cell death via protein degradation. As such, Talamantez-Lyburn et al. ${ }^{[104]}$ developed a method for the delivery of Cul5 DNA to cells employing AuNPs. The nanocarriers were able to increase the sensitivity of Cul5 deficient AU565 cells, providing evidence that AuNPs-assisted delivery of DNA can easily lead to the sensitization of drug-resistant tumor cells.

\section{Silver nanoparticles}

Silver nanoparticles (AgNP) have been extensively used in the treatment of MDR cancer, with a great dependency of the efficacy on the size of the nanostructures. Still, there is an important window for research that would allow targeting some of the deadliest drug-resistant tumors.

As such, a relevant example was shown by Gopisetty et al. ${ }^{[105]}$, who decided to examine AgNPs sizedependent cellular features in MCF-7/KCR cells. They reported that $75 \mathrm{~nm}$ AgNPs were more efficient in inhibiting the P-glycoprotein (Pgp) efflux activity in the MCF-7/KCR cells (thus potentiating the apoptotic effect of DOX) than the $5 \mathrm{~nm}$ nanoparticles. Meanwhile, the $5 \mathrm{~nm}$ AgNPs were more effective ROS producers, most likely due to a higher surface-to-volume ratio. It was observed that the $75 \mathrm{~nm}$ AgNPs were able to affect the endoplasmic reticulum calcium stores, thus causing the ER stress, and decreasing the plasma membrane positioning of Pgp. This was regarded as a determining mechanism of Pgp inhibition, which was proven effective in sensitizing the multi-drug resistant breast adenocarcinoma towards DOX treatment. Therefore, the $75 \mathrm{~nm}$ AgNPs can be used as powerful inhibitors of Pgp function and are promising agents for sensitizing multidrug-resistant cancers to anticancer drugs.

In a similar study, AgNPs were used to sensitize the A2780-Pt cells towards Pt treatment. Cur-coated AgNPs were biologically synthesized. Synergic cellular effects of AgNPs and Pt on the cells were assessed. Results showed that 8 and $62 \mu \mathrm{g} / \mathrm{mL}$ of AgNPs and Pt led to 50\% cell death in $48 \mathrm{~h}^{[106]}$. Similarly, AgNPs were shown to exert an inhibitory action on the efflux activity of MDR cancer cells, when the synergistic interactions of AgNPs with six different antineoplastic agents on drug-resistant cells were tested ${ }^{[107]}$.

Overall, the use of metal nanoparticles in the treatment of drug-resistant cancers has been widely researched. The surface areas of metal nanoparticles, such as gold and silver nanoparticles, allow for the attachment of bioactive molecules, e.g., antibodies, that help to sensitize the cancer cells,. Furthermore, other chemical properties, such as hydrophilicity and stability, also contribute to effective drug delivery. However, it has been proven that the viability of metal nanoparticles is highly dependent on their size and, to some extent also shape. Thus, there is much more research to be done to improve metal nanoparticles for more effective anticancer treatment.

\section{Magnetic nanocarriers}

One of the biggest advantages of magnetic nanoparticles (MNPs) - compared with non-magnetic materials - is the possibility to be controlled by an external magnetic field. In the case of nanocarriers, this feature may support particle guidance, uptake, and accumulation at intended destinations, especially in combination with another targeting agent. For example, Wang et al. ${ }^{[108]}$ modified yolk-shell Fe3O4@ $\mathrm{MgSiO} 3$ nanoparticles with polyethylene glycol conjugated with folic acid (FA), which binds folate receptors overexpressed in various cancerous cells ${ }^{[109]}$. An in vitro study on drug-resistant GEP-G2/MDR cells confirmed the internalization of these NPs via the protein-independent endocytosis pathway that obviated mechanisms typically associated with a DOX resistance. Moreover, synergism between FA and magnet-assisted targeting resulted in increased accumulation of the particles in the Hep-G2/MDR tumor xenografts in mice models and effectively inhibited tumor growth. 
In turn, Cho et al ${ }^{[110]}$ developed magnetic tandem apoptosis triggers (m-TATs), consisting of the magnetic core, targeting monoclonal antibodies, and DOX. Exposure to a static, low-gradient magnetic field facilitated cellular internalization of particles and simultaneously induced their clustering that activated the extrinsic apoptosis cascade process. The synergism of action between m-TAT components resulted in absolute mortality of the DLD-1/ADR colon cancer cell line in vitro and complete retraction of the tumor in vivo in the xenograft mice model. Instead of using whole antibodies, Truffi et al. ${ }^{[111]}$ modified MNPs with multiple half chains of anti-HER2 monoclonal antibodies, Tmab. The potential of these MNPs was tested on various cells, including Tmab-resistant BT474TR and JIM1-4 cells. The particle concentration of $0.2 \mu \mathrm{g}$ mL-1 was enough to accumulate in $100 \%$ and $40 \%$ of BT474TR and JIM1-4 cells, respectively. However, the additive effect on Tmab-conjugated particles and DOX on cell viability was observed only in BT474TR cells.

Cell-nanoparticle interactions can also be enhanced by the modification of MNPs with peptides. MillerKleinhenz et al. ${ }^{[112]}$ developed a peptide with an ability to bind and inhibit simultaneously the Wnt-related LRP5/6 and urokinase plasminogen activator (UPAR) receptors, which was subsequently conjugated with DOX-modified MNPs. This strategy limited cell invasion in MDA-MB-231 via newly recognized mechanisms associated with Axin and $\beta$-catenin regulation but did not affect cell viability in vitro. An in vivo study on the orthotopic human chemo-resistant breast cancer patient-derived xenograft mouse model showed combinatorial effects of chemotherapy and inhibition of the Wnt/beta-catenin signaling pathways, resulting in a significantly reduced number of proliferating cells and minimized average tumor volume.

Furthermore, Liu et al. ${ }^{[113]}$ and Weng et al ${ }^{[114]}$ exploited cell-penetrating peptides to deliver Ag-Fe3O4 with DOX and Fe3O4 loaded with Pt, respectively. The proposed NPs were characterized not only by an enhanced internalization but also showed unexpected ability - mediated by ROS produced via the Fenton reaction - to reverse drug-resistance in HNE-1/DDP and CNE-2 cells. The high toxicity of ROS was also employed by Ma et al. ${ }^{[115]}$ to enhance the anticancer activity of the Pt prodrug conjugated with FePt NPs - iron. In this study, the release of ROS from the NPs induced mitochondria dysfunction, activating the apoptotic cascade and sensitizing drug-resistant ACP cells to Pt. The magnetic field applied in vivo (tumorbearing mice model) supported the accumulation of the particles in the tumor, contributing to significantly increased ROS levels and greater inhibition of tumor relative volume after 14 days, compared to free Pt.

Enhanced ROS production in cancer cells was also achieved by loading multi-core MNPs with an artemisinin derivative as proposed by Guo et al. ${ }^{[16]}$. In this study, the dose-dependent toxicity of the dihydroartemisinin-MNPs complex, superior to those observed for DOX, was proven against both MCF-7 and MCF-7/ADR cells. This was assigned to the release of ferrous ions from MNPs under acidic conditions, which further catalyzed the DHA-related ROS production. In contrast, Yen et al. ${ }^{[117]}$ conjugated MNPs with catalase - an enzyme converting ROS to molecular oxygen - to mitigate hypoxic environments in tumors as insufficient oxygen supply activates the production of hypoxia-inducible factors participating in the development of multidrug resistance. Catalase-modified MNPs not only maintained the $\mathrm{pH}$ dependent profile of the enzyme activity, prolonging it 3-fold, but also showed significantly higher toxicity in combination with PTX towards drug-resistant 4T1 cells compared to both free PTX and catalase+PTX treatments.

Many phenolic compounds, famous for their anti-oxidant and anti-inflammatory properties, also showed anti-cancer activity and thus can be used as chemosensitizers ${ }^{[118]}$. Among various phenolic compounds, Cur is one of the most widely exploited, acting as a chemodepressor for co-delivering cytostatics using magnetic nanocarriers ${ }^{[119-122]}$. Rastegar et al. ${ }^{[123]}$ developed MNPs modified with hydroxyapatite and $\beta$-cyclodextrin, which provided effective encapsulation of DOX and Cur that resulted in significantly reduced cell viability of MCF-7 and MCF-7/ADR cells, compared to free DOX. Even though the magnetic guidance facilitated 
the accumulation of these particles at the targeted site in $4 \mathrm{~T} 1$ tumor-bearing mice, the substantial impact of co-delivery on relative tumor volume was not observed in this study. However, the P-gp expression analysis confirmed remarkable Cur-mediated inhibition of the efflux pump.

Quercetin (QUR) was also used to overcome drug-resistance in cancerous cells. Daglioglu ${ }^{[124]}$ developed pH-sensitive MNPs conjugated simultaneously with QUR and DOX that showed an ability to significantly increase the effect of DOX on A546/DOX by augmentation of the G2/M-phase cell cycle arrest leading to reduced cell proliferation and increased apoptosis ${ }^{[124]}$. Besides, this nanosystem showed a limited impact on BEAS- $2 \mathrm{~B}$ cells, which proved that this nanosystem has limited cytotoxicity towards non-cancerous cells.

MNPs were also used to load different types of nanocarriers to enable their control via the magnetic field. Wang et al. ${ }^{[125]}$ reported mesoporous silica-based NPs loaded with MNPs for dual chemo/photodynamic therapy, which effectively reversed resistance and induced apoptosis in cancer cells in a dose-dependent manner. The exposure to a magnetic field increased the cellular uptake of the NPs in drug-resistant cells, as well as significantly enhanced their retention in the tumor in vivo, which contributed to the prolonged mean survival duration of MCF-7/ADR tumor-bearing mice. Additionally, in a study by Wang et al. ${ }^{[119]}$, loading of polymeric particles, made of biotin-poly(ethylene glycol)-poly(Cur-dithio dipropionic acid), with MNPs contributed to significant enhancement in the cellular uptake of the nanosystem by MCF-7/ ADR cells upon the magnetic field, which resulted in a 2 -fold higher resistance reversion index against cancerous cells, as compared to the non-magnetic-assisted treatment.

Overall, MNPs represent viable methods of the treatment for chemoresistant cancers because they can be controlled by external magnetic fields, which can assist uptake and accumulation of nanocarriers at intended destinations. Many studies have been done to determine the function of MNPs in the treatment of chemo/drug-resistant cancers. MNPs present modified ROS that aid in the reversal of drug resistance in cancer. However, MNPs can present a viability problem with a certain cell line. Thus, more consistent research must be performed with MNPs.

\section{Carbon-based nanocarriers}

Carbon nanomaterials (CNMs) are reported to produce safe and effective drug delivery systems for targeting therapy-immune cancer cells. CNMs include nanodiamonds (NDs) in $\mathrm{sp}^{3}$ hybridization, graphene/graphene oxides (GO), and carbon quantum dots (CD) in $\mathrm{sp}^{2}$ hybridization, carbon nanotubes (CNTs), and fullerenes in $\mathrm{sp}^{2-3}$ hybridization ${ }^{[126,127]}$, the atomic structure of which is depicted in Figure 7.

Among these, graphene, CNTs, and carbon dots are the most commonly used, and this is due to two unique qualities. Firstly, they possess a large share of delocalized $\pi$ bonds able to bond hydrophobic drugs by $\pi$ stacking ${ }^{[128,129]}$. Secondly, they have unique optical properties, such as near-infrared absorption and emission, which allow their usage for imaging and photothermal therapy. Other favorable qualities shared for some CNMs are listed in Figure 8. These properties allow for fabricating multifunctional delivery systems with the possibility of loading more than one cargo onto a single molecule and, at the same time, that enable real-time tracking of NMs distribution by fluorescence, and allow for photothermal (PTT) or photodynamic therapy (PDT, as a photosensitizer and photosensitizer carrier) for an effective theragnostic approach $^{[130,131]}$.

The possibility of loading multiple cargos arises from the CNMs' unique intrinsic properties; cargo can be filled inside the material's cavity ("endohedral filling"). Also, other factors, such as interactions with the CNMs' surface (electrostatic, click chemistry, covalent ${ }^{[132,133]}$ ) and $\pi-\pi$ stacking, contribute to the transport of additional materials ${ }^{[134]}$. By this means, due to an extremely high surface area, high amounts of various molecules could be bonded to the same particle. These cargos can be quickly released in the acidic 

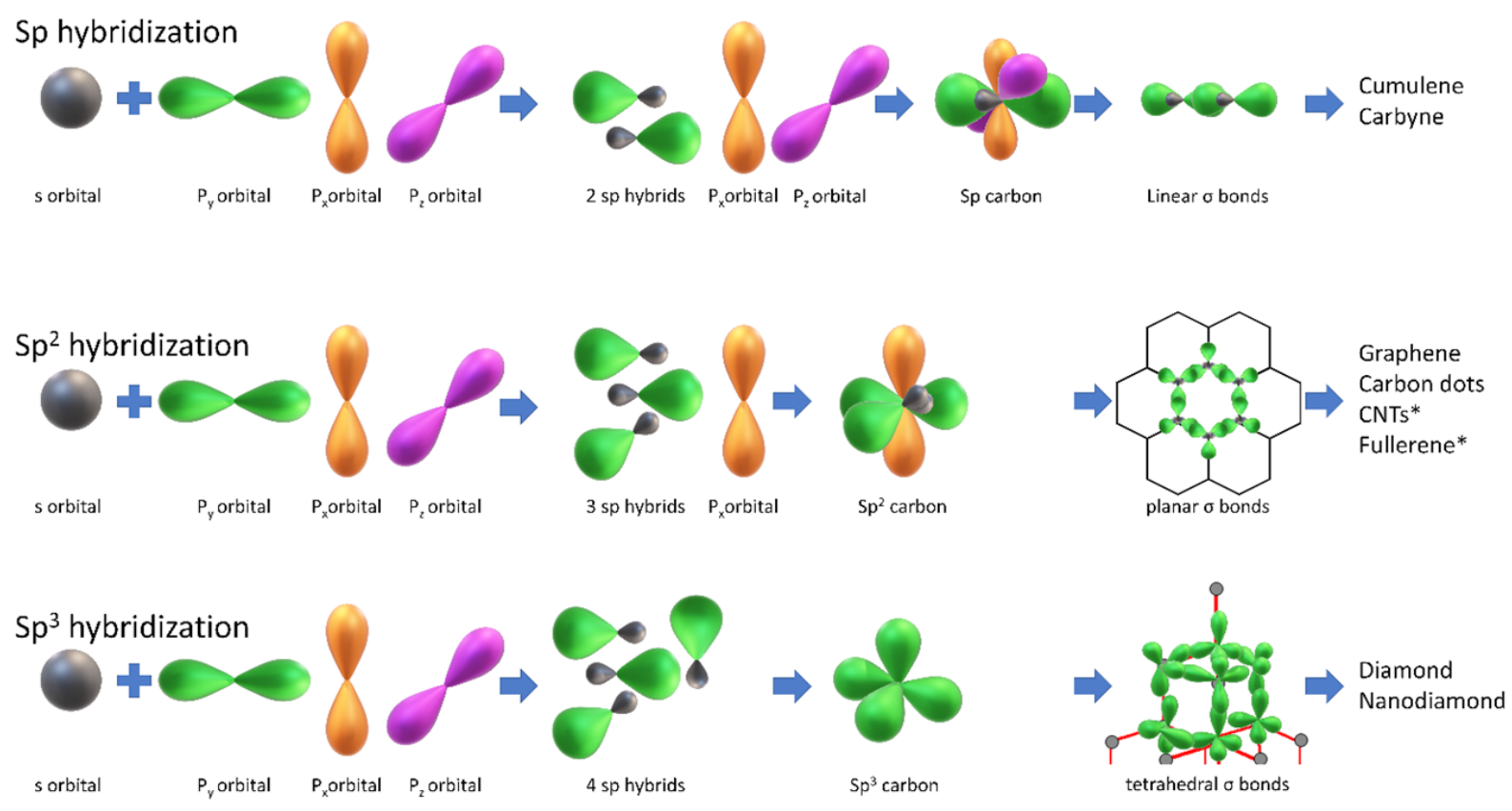

Figure 7. Schematic representation of various hybridizations of carbon, together with their corresponding allotropes. Note, for simplification, CNTs and Fullerenes are presented as sp ${ }^{2}$ carbons. ${ }^{*}$ Denotes transitional hybridization between 2-3

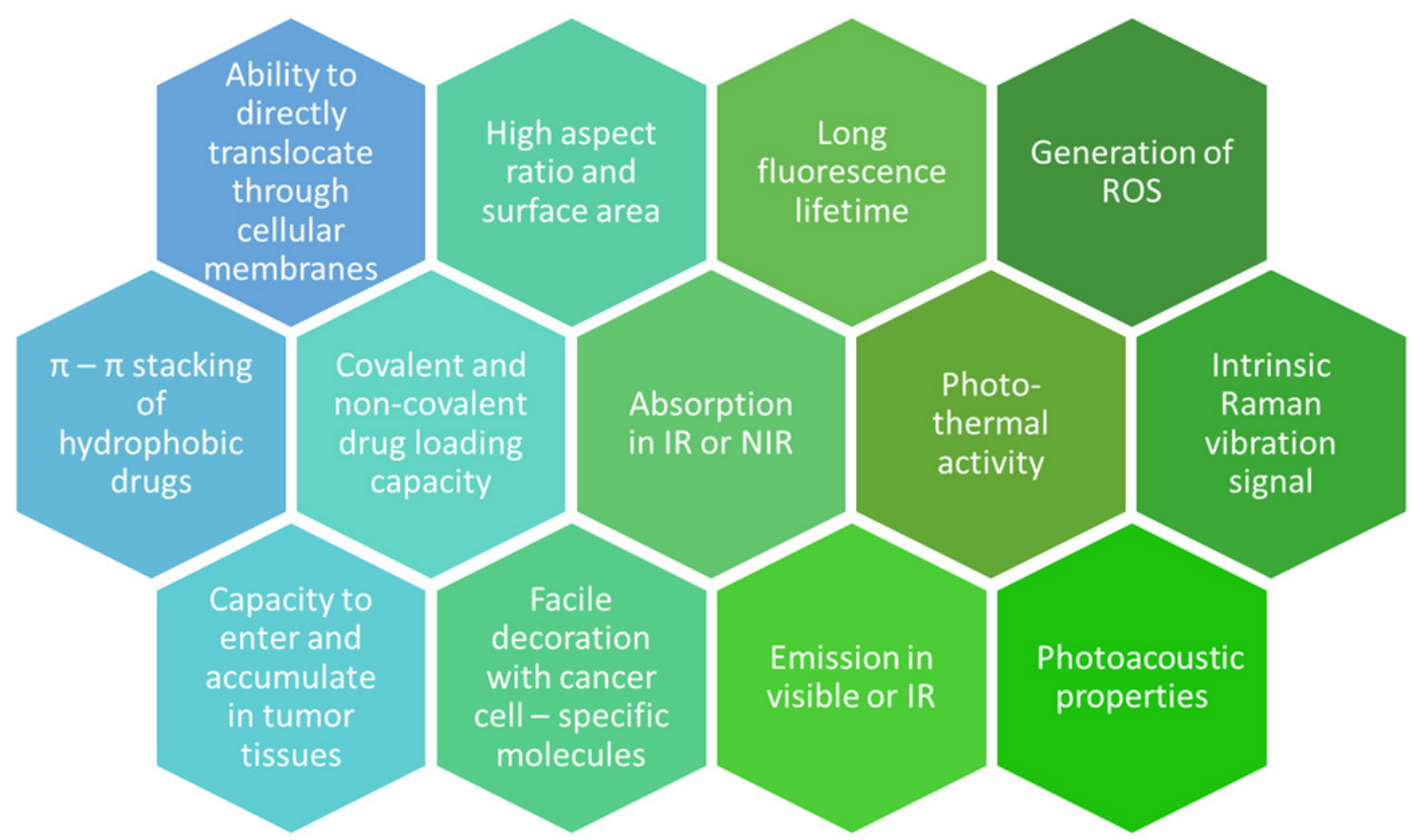

Figure 8. Selected properties of carbon-based nanomaterials that constitute for their popularity in the field of nanomedicine-based anticancer therapy ${ }^{[141,144,155,]}$

environments of endosomes and lysosomes of cancer cells that can cleave the drug-CNMs interactions ${ }^{[135]}$. Examples of this approach are visualized in Figure 9, and these can be divided by the nature of the bonded species: 


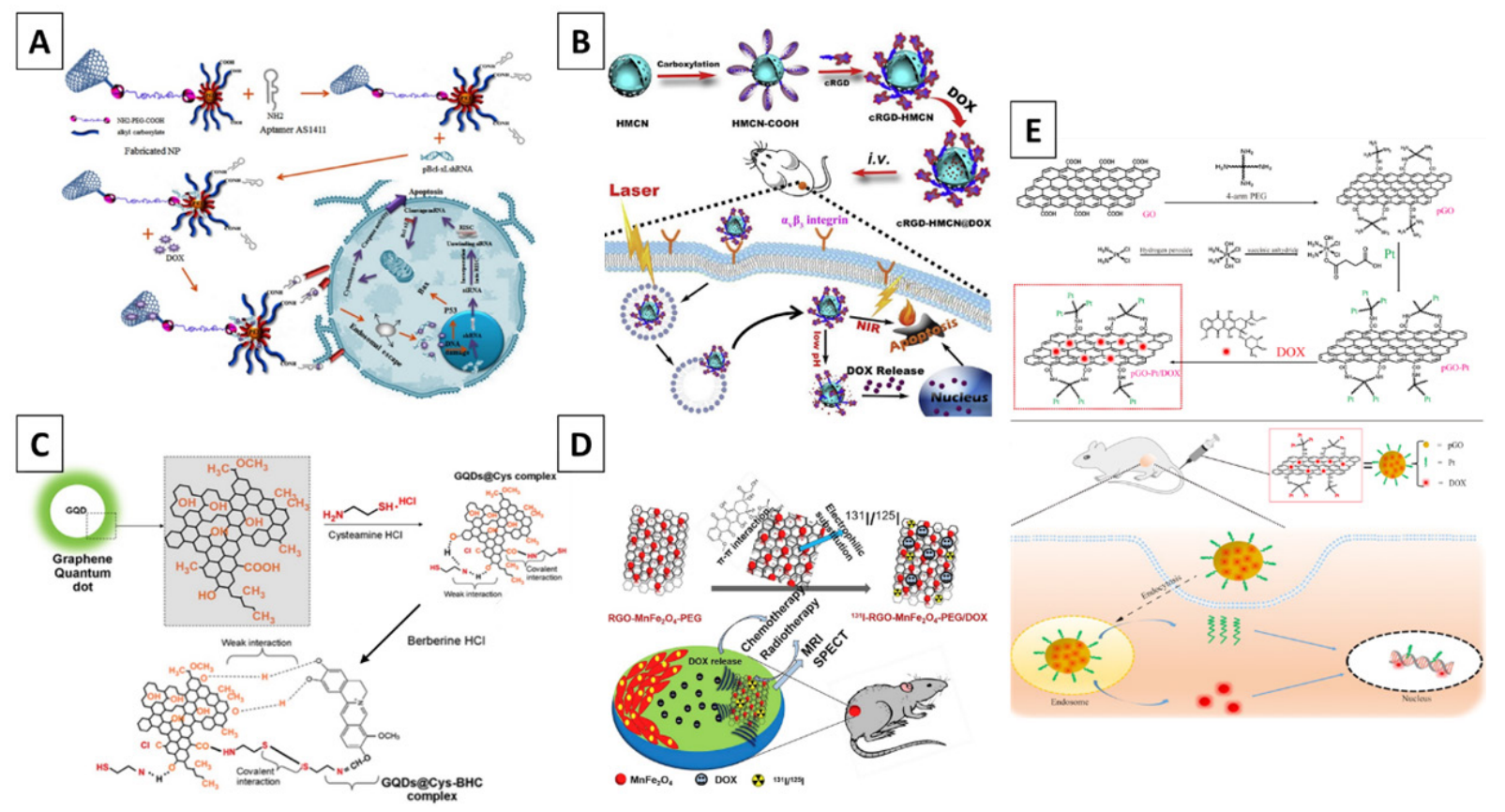

Figure 9. Examples of strategies used to bond more than one bioactive molecule to the CNMs. Covalent bonding of polyethylenimine and

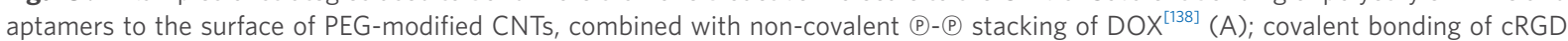
combined with the loading of DOX into the CNMs' cavity for selective targeting of cancer cells ${ }^{[135]}(\mathrm{B})$; combination of covalent and noncovalent binding of a single drug for the controllable release from graphene quantum $\operatorname{dots}^{[167]}(\mathrm{C})$; manganese ferrite grown on the surface of graphene oxide, followed by modification with a radioisotope via electrophilic substitution and $\left(\odot-\oplus\right.$ stacking of DOX ${ }^{[143]}$ (D); two anticancer drugs, bonded to the surface of graphene via covalent (Pt) and non-covalent (DOX) interactions for an enhanced anticancer effect $^{[142]}(E)$

1. Hydrophobic drugs (such as DOX, SN38, and PTX) can be bonded by the $\pi-\pi$ stacking mechanism for improved dispersion and facilitated release of those drugs ${ }^{[136-138]}$;

2. Different targeting molecules for selective delivery to cancer cells can be attached via click chemistry or covalent modification. Some examples are: (a) ferritin ${ }^{[139,140]}$ and $\operatorname{transferrin}^{141}$, both of which bind to cells via the transferrin receptor 1, overexpression in cancerous cells; (b) iRGD or cRGD for targeting the avb3integrin of tumor endothelium; and (c) candesartan (CD) for targeting AT1R in lung cancer cells ${ }^{[141,142]}$;

3. Biological compounds, such as peptides, proteins, nucleic acid, etc. are attached via covalent interactions, click chemistry, or electrostatic attraction, enabling improved solubility, biocompatibility, and increased protection from uncontrolled cleavage and degradation ${ }^{[136,137]}$;

4. Magnetic and ferromagnetic particles can be entrapped within the materials cavity or bonded to their surface for magnetic field-assisted selective delivery and enhanced contrast for magnetic resonance imaging ${ }^{[143]}$.

CNMs can have various sizes that can be comparable to natural compounds: proteins $(1-100 \mathrm{~nm})$ and DNA (2-3nm wide $)^{[144]}$. They can enter the cells through passive diffusion (also referred to as needlelike penetration, mainly reported for $\mathrm{CNTs})^{[145]}$ or various adenosine triphosphate (ATP)-dependent uptake mechanisms (dynamin-dependent) ${ }^{[146]}$. The leading uptake mechanism depends on the CNMs' physicochemical properties and the type of the cell ${ }^{[145]}$.

Due to the high popularity of CNMs in the cancer theragnostic, many articles on the matter can be found, and additional background information can be found in these excellent articles ${ }^{[130,141,144,147-155]}$. Additionally, information about how CNMs' intrinsic properties can serve in theragnostic approaches [PTT and PDT, optical imaging, and cargo delivery systems (including stimuli-responsive and cancer cell-selective)] and 
how to obtain biocompatible CNMs can be found in these sources. By combining different cancer-targeting strategies and facilitating selective intake and accumulation in cancer cells, CNMs can be regarded as effective tools for fighting chemoresistance in cancer cells. Most notably, it has been reported that CNMs-based PTT can reverse the resistance of cancer cells to a certain drug and target cancer stem cells responsible for remission ${ }^{[156]}$. In the following sections, examples of some of the most interesting and recent approaches in targeting cancer cells using various CNMs based cargo-delivery systems are given. These are grouped by the type of CNM.

\section{Carbon nanoparticles}

Carbon nanoparticles are spherical nanomaterials, typically made up of amorphous carbon, with varying amounts of graphitic planes. The presence of the latter allows for $\pi-\pi$ stacking of hydrophobic drugs and grants the materials with excitation dependence - fluorescence, typically in the NIR-I window ${ }^{[157]}$. Most often, these materials will possess a hollow core, into which a magnetic particle can be loaded ${ }^{[158]}$, and a mesoporous structure, useful to load bioactive molecules ${ }^{[135]}$. Oxidation can be used to introduce functional groups for enhanced dispersion and covalent interactions with drugs ${ }^{[157]}$.

Depending on the synthesis method, the sizes of the CNPs can range from a few nanometers to a few hundred nanometers, enabling various internalization pathways. In cancer drug delivery, the CNPs are most typically loaded with hydrophobic drugs, and the therapeutic effect can be boosted by gene or thermal therapy. In a study by Zhao et al. ${ }^{[159]}$, the morphology dependent performance of CNPs was tested, and it was found that spherical, rough, and hollow particles are superior in employing a combined gene/PT/ chemotherapy able to kill over $90 \%$ of a mice analog of metastatic, chemoresistant stage IV breast cancer cells, both in vitro and in vivo. Remarkably, the authors had managed to fabricate materials with the NIR-II fluorescence window for effective PT. This region is favorable as it yields a more effective emission, even in deep tissue, due to reduced scattering ${ }^{[144]}$.

In a different approach, studied by Fan et al. ${ }^{[139]}$, the CNPs were found to produce a cancer cell-killing effect without the usage of an external bioactive molecule. Conjugation with hollow human H-ferritin NPs yielded a selective and effective material that entered the cells via clathrin-dependent endocytosis, localized in lysosomes, and caused ROS burst and hypoxia. Intravenous injection in vivo resulted in completely arrested hepatocellular carcinoma and colon cancer tumor growth at day 5 , with mortality reduced to zero and a complete recovery reported in $40 \%$ cases, and no damage to vital organs observed. The particles were safely cleared through urine and feces. These remarkable results should be treated as a guide for the future development of effective, nanomaterial-guided cancer eradication. Because no drug was used, it should be hypothesized that this strategy would be effective in treating chemoresistant cancers. However, further evaluation should be performed to confirm such supposition.

Other teams have also reported successful and selective eradication of chemoresistant, metastatic cancers in vivo, by enabling the delivery of DOX and $\mathrm{CPT}^{[135,160-162]}$. In a study by Li et al ${ }^{[161]}$, mesoporous carbon nanospheres were loaded with DOX and coated with PEG - poly(Cur-dithiodipropionic acid) (PEGPCDA). The latter was used to grant the materials with improved dispersibility and provide them with chemosensitizing quality. Upon entering the cells, glutathione of the cytoplasm cleaved the bonds in the (PEG-PCDA), leading to Cur release, which increases the drug accumulation in the cancer cells. After the shell of the CNM was removed, the $\mathrm{pH}$-sensitive release of DOX was able to take place. The obtained system was used to kill chemoresistant MCF-7/ADR cells effectively. Still, further studies are needed to evaluate whether or not the system can be safely used - in vitro studies to establish cytotoxicity against normal and healthy cells, followed by in vivo evaluation to determine the long-term effect and fate of the CNMs in the living organism. 


\section{Carbon dots}

The term carbon dots (CDs) most typically refers to small fragments (usually a few $\mathrm{nm}$ ) of graphene planes, cut down via chemical or physical treatments. To avoid agglomeration, oxygen atoms are introduced into the structure. CDs combine features characteristic of graphene - NIR fluorescence, PT effect - with simpler internalization and the ability to be eliminated from the body via urinary clearance.

In the literature that regards nanomedicines against cancer, CDs are typically used for better tumor visualization, in PTT, and for fabricating $\mathrm{pH}$-sensitive drug delivery systems ${ }^{[163,164]}$. Selective accumulation is achieved by the EPR effect ${ }^{[165]}$ or through specific modification, e.g., by folic acid ${ }^{[166]}$. In the study against stage IV metastatic breast cancer cells, CDs were able to deliver BHC drugs inside the cells, inducing apoptosis in $99 \%$ of the culture and granting the ability to visualize the cells via fluorescence $e^{[167]}$. In a different study, Feng et al. ${ }^{[164]}$ modified the CDs with Pt and DOX using $\mathrm{pH}$ cleavable covalent bonds. The materials were easily internalized by Pt-resistant human ovarian cancer cells, enabling fluorescent imaging. Under the acidic $\mathrm{pH}$ of cancer cells, a controllable release of drugs resulted in the efficient killing of cancer cells at significantly lower concentrations than the Pt alone. Unfortunately, an effect on the healthy cells was not evaluated, and the materials were not subjected to extensive in vitro or in vivo evaluation. A more comprehensive evaluation was done by Sui et al. ${ }^{[168]}$, who proposed a drug delivery system able to selectively attack cancer cells and sensitize Pt resistant cancer for effective chemotherapy treatment. No adverse effect on healthy cells was observed, both in vitro and in vivo.

\section{Nanodiamonds}

Nanodiamonds are nanoscaled materials (typically up to tens of $\mathrm{nm}$ ), composed mainly of carbon atoms in the $\mathrm{sp}^{3}$ hybridization (tetrahedral). For such materials to exist as stand-alone structures, the outermost atoms need to be capped. This is done either by heteroatoms or by carbon atoms in different hybridizations. As such, NDs are open for functionalization strategies that can anchor different molecules. Additionally, the existence of specific defects in the structure can grant the NDs with photoluminescence, enabling PTT. These features are highly desired in nanomedicine ${ }^{[144,169,170]}$. In a study by Yu et al. ${ }^{[171]}$, the performance of NDs in delivering PTX into chemoresistant cells was compared with a commercially available NPs-based system, Abraxane. It was found that the particles readily accumulated in lysosomes, and the efficiency of killing cancer cells was significantly improved as compared to Abraxane (71\% cells were killed as compared to $40 \%$ upon $48 \mathrm{~h}$ culture). This clearly indicated the superior performance of the NDs in this field, most likely attributed to better uptake and $\mathrm{pH}$-sensitive drug release.

NDs have also been reported to successfully deliver drugs used to fight chemoresistance in cancer cells by blocking the P-glycoprotein from pumping-out, which is over-expressed in multi-drug resistant cancer cells and participates in the clearance of some of the anticancer drugs, such as DOX or Pt. In a study by Zhu et al. ${ }^{[172]}$, a double drug loading of NDs with a physisorbed malaridine (MAL) and DOX, followed by covering with folate-DOX-PEG, was done. The resultant material was easily dispersed, readily entered the cells, and revealed a pH-responsive cargo release. The presence of MAL increased the treatment efficiency against chemo-resistant MCF-7/ADR cells by improving the cellular accumulation of DOX. In in vitro studies, apoptosis was induced in over $90 \%$ of cells, proving the high efficiency of this combinatorial approach. However, further studies need to be done to prove the system's safety in vivo. On the other hand, Lam et al. ${ }^{[173]}$ has shown that NDs by themselves can also be used to reduce the P-glycoprotein efflux. By covering the NDs with either GEF or erlotinib (EL), a promising therapeutic effect was achieved.

The efficiency of the anti-cancer treatment can also be increased by introducing specific targeting molecules. This avenue was investigated by Chan et al. ${ }^{[17]}$. Selective targeting of cancer cells over healthy cells was achieved by introducing folic acid (FA) moieties. To enhance the performance even further, a mitochondrial localizing sequence (MLS) was used to favor drug accumulation in mitochondria over 
lysozymes. As a consequence, a superior system that was safe to healthy cells but detrimental to cancer was obtained $^{[174]}$.

In a different study by Li et al. ${ }^{[175]}$, NDs were used to fabricate an efficient glioblastoma-targeted treatment. Glioblastoma is a highly malignant brain cancer that has very mortality due to chemoresistance, lack of effective treatments, and the inability of pharmaceutics to cross the blood-brain barrier (BBB). In the study, NDs modified with DOX were taken up by dendritic cells (DC), which were used to deliver a payload through $\mathrm{BBB}$ and into glioblastoma cells (GC). As a result, adjuvanticity and antigenicity of the GC cells were stimulated, which, in turn, promoted maturation of DC and initiation of an immune response via $\mathrm{T}$ lymphocytes. Thus, the material was suggested as a novel tool able to subvert the glioblastoma immunosuppressive microenvironment and to employ anti-GBM immunotherapy. Thus, a selective accumulation in the brain was achieved, indicating that the therapy should be safe and not affecting vital organs ${ }^{[175]}$. In the follow-up studies, the mechanism of the NDs-related action was attributed to the activation of autophagy ${ }^{[176,177]}$. In a different study by the same team, the NDs-DOX complex was found to suppress the glioblastoma - astrocyte cross-talk, breaking the chain responsible for acquiring resistance towards various therapies by evading apoptosis ${ }^{[178]}$. The same material was also used to evade chemoresistance and reverse cancer-induced immunosuppression in triple-negative breast cancer ${ }^{[179]}$. Thus, the NDs can be suggested as one of the most promising nanomaterials for the treatment of therapyresistant cancers.

\section{Graphene and graphene oxide}

Graphene is a $2-\mathrm{D}$ material made up of a single honeycomb lattice. This material has roughly a 2-times higher surface area than CNTs (suitable for drug delivery) and better electrical properties. Fluorescence is observed from the ultraviolet (UV) to near-infrared (NIR). Heat can be emitted upon absorption of light or acoustic waves, enabling PTT or thermoacoustic therapy. Selective imaging with better contrast can be achieved via fluorescence, photothermal, photoacoustic, and Raman scattering effects. Thus, graphene possesses a set of properties, making it suitable to be used in novel anti-cancer therapies. However, one limitation lies in the fact that it contains a high amount of unlocalized $\pi$ electrons, causing a spontaneous aggregation of graphene planes, reducing the material's applicability. Hence, its oxidized form - $\mathrm{GO}^{[144]}$ - is the most typically employed.

GO has been successfully used in targeting different types of tumors, including metastatic and chemoresistant cancers. In a study by Pei et al. ${ }^{[142]}$, the combination of two chemotherapeutic drugs, PTX and DOX, delivered into the cells by GO resulted in an effective eradication of cancers, both in vitro and in vivo, without adverse side effects. Remarkably, CNMs were able to reduce the size of highly chemoresistant tumors by over $60 \%$. A similar approach of combining more than one drug was also employed by Tiwari et al. ${ }^{[180]}$ and Tran et al. ${ }^{[181]}$ with similarly promising results.

In a study by Thapa et al. ${ }^{[182]}$, DOX was combined with RAPA, an inhibitor of phosphoinositide-3-kinase (PI3K)/Akt/mechanistic target of rapamycin (mTOR) cell survival pathways, one of the many mechanisms of resistance development. By combining the material with PTT, nearly $80 \%$ of chemoresistant cancer cells were killed in vitro. Certainly, further studies on the safety and in vivo efficiency of the system are needed. In different studies, modification with drug targeting molecules, such as lactobionic acid ${ }^{[183]}$, hypericin ${ }^{[184]}$, prostate stem cell antigen (PSCA) monoclonal antibody $\left(\mathrm{mAb}_{\mathrm{psca}}{ }^{[185]}\right.$ or hyaluronic acid ${ }^{[186]}$ had resulted in a more selective and effective eradication of cancer, reaching up to $80 \%$ killing efficiency via induction of apoptosis. Interestingly, in the study by Guo et al. ${ }^{[185]}$, additional modification with magnetic particles allowed for imaging of tumors with high resolution and contrast. The cancer-killing efficiency can be further improved by employing $\mathrm{PT}^{[181,182]}$ or radiotherapy ${ }^{[143]}$, with tumor cell viability reduced to up to $90 \%$. 
Therefore, it can be concluded that effective killing of chemoresistant cancer cells can be achieved by employing GO as a carrier combined with multimodal therapies, such as multiple drug usage, radiotherapy, PTT, and employing cancer-targeting molecules. These therapies sensitize the cells towards further treatment, enabling the effective delivery of higher doses of therapeutic agents into the cancer cells while minimizing the risk of adverse side effects.

\section{Carbon nanotubes}

Carbon nanotubes (CNTs) are composed of cylindrically rolled-up graphene planes, either single (SWCNTs) or multiple (MWCNTs). The typical diameter of SWCNTs is below $10 \mathrm{~nm}$, which helps facilitate passive transport into the cells; while, at the same time, results in higher cytotoxicity. Meanwhile, MWCNTs with diameters exceeding $15 \mathrm{~nm}$ are usually reported to be less toxic ${ }^{[187]}$. In both cases, reduced lengths contribute to reduced toxicity, both in vitro and in vivo. Regardless of the administration route and hydrophilicity, dispersible materials generally have better applications ${ }^{[188]}$. Depending on the rolling axis, three different conformations of SWCNTs can be obtained, which determine electrical and optical properties, such as arm-chair (metallic), chiral, or zig-zag (semi-conducting). From the cancer drug delivery point of view, semiconducting SWCNTs are beneficial as they present photoluminescence in the NIR-II window upon excitation, enabling imaging of deep tissues with good contrast ${ }^{[144]}$. MWCNTs usually present metallic conductivity and, in some cases, exhibit photoluminescence and photothermal properties $^{[156,189]}$.

As a literature survey reveals, MWCNTs are more often applied in fighting chemoresistant cancer than SWCNTs, which is probably due to higher uniformity of electrical and optical properties and better biocompatibility. With encapsulation efficiency usually exceeding 50\%, internalization via endocytosis, and sustained linear drug release upon cellular internalization, these materials seem to be very promising candidates as novel therapies against chemoresistant cancers. In a series of studies by Dong et al. ${ }^{[156,189]}$, oxidized MWCNTs were $\pi-\pi$ stacked with DOX and modified with TAT-chitosan conjugate to fabricate a biocompatible platform for the sustained release of the drug. Intratumoral administration into mice bearing BEL-7402 xenograft tumors resulted in an effective reduction of tumor size for improved survival rates by combining PTT with drug-related effects. Raza et al. ${ }^{[190]}$ had used oxidized MWCNTs to bind two types of bioactive molecules via different bonds. The results indicated that the obtained nanomedicine is biocompatible and able to target cancer cells. This resulted in sensitized chemoresistant MDA-MB-231 cells. In a study by Zhang et al. ${ }^{[191]}$, MWCNTs were covalently bonded with gadolinium-conjugated CDs and $\pi-\pi$ stacked with DOX, followed by modification with an EGFR antibody. The materials were selectively uptaken by A549 cells and metastatic MDA-MB-231 cells, and exhibited pH and NIR-responsive drug release, with a photothermal effect. In in vivo experiments, A549 tumor-bearing mice, subjected to intratumoral injections and PTT, revealed a $100 \%$ tumor elimination. Sadly, as in vivo studies were not performed on chemoresistant cancers and the intratumoral administration route is not often clinically applied, it is hard to evaluate the actual value and the clinical transferability of the presented data. Certainly, further studies are needed.

Safe and successful usage of SWCNTs in eradicating cancer cells has also been reported. Most notably, in a series of studies by Guven et al. ${ }^{[192,193]}$, the ability to fabricate highly selective nanomedicine that can be delivered and released in a controlled matter was found. For example, a highly potent and hydrophobic drug, Pt, with the efficient killing of cancer cells, was created. The accumulation of NMs in cancer cells was attributed to the EPR effect. In a different study by Ghosh et al. ${ }^{[194]}$, estradiol positive (EP) cancers were targeted by modifying SWCNTs with $17 \beta$-estradiol based amphiphiles, which resulted in the selective killing of ca. $70 \%$ of EP cancers. While CNTs are most typically suggested as an efficient tool used to bind hydrophobic drugs for improved administration efficiency, a completely different approach was suggested by Razzazan et al. ${ }^{[195]}$. In this study, binding with CNTs was used to improve retention of a hydrophilic drug 
(GEM), increasing its resistance towards enzymatic attack and improving its plasma half-life. At the same time, efficient killing of cancer cells, such as, MIA PaCa-2, pancreatic cancer cells, which have a significant tendency of developing drug resistance via epithelial-mesenchymal transition, was achieved in vitro. In vivo, a selective delivery was proven, with smart, $\mathrm{pH}$-dependent drug release. As a result, tumor growth was significantly arrested, with one case of complete recovery reported.

\section{Summary}

In summary, carbon-based nanomaterials can be regarded as one of the most promising nanomaterials to be applied in nanomedicine-aided anti-cancer therapy. The ability of these nanomaterials to bind multiple molecules in conjunction with the employment of PTT, PDT, and other complementary techniques has proven to sensitize drug-resistant cancers and provide multiple cell-killing strategies for effective eradication of cancer, both in vitro and in vivo [Figure 10]. CNMs have also proven the ability to provide cancer-selective vehicles with no detrimental effect observed towards healthy cells or living organisms. The selectivity can be achieved via the EPR effect, functionalization with target specific molecules, or modification with magnetic particles. However, when considering CNMs-based cancer-killing strategies, strict caution needs to be applied - these materials are known to induce varying reactions towards healthy cells and organisms, strictly depending on their physicochemical properties. A broad spectrum from complete biocompatibility to severe toxicity or tumorigenicity is covered. This phenomenon is the main reason for high discrepancies and controversies regarding biomedical usage of CNMs, unlike other materials cited in this study that are, in general, intrinsically biocompatible.

\section{CONCLUSION AND FUTURE REMARKS}

Over the past few decades, many cancers have been reported to show some variable degree of drug resistance to the usual treatments, a reality that is craving for new ways to treat these tumors. There are two categories of factors that are responsible for drug resistance: intrinsic and extrinsic. Drug-resistance in cancer cells prevents the onset of cell death, autophagy, and apoptosis. Thus, new methods are being developed for the treatment of resistant cancer incorporating the use of nanomaterials, as discussed in this review. Nanomaterials can be designed for passive or active targeting of cancer cells, and they can help in transport, delivery, and release therapeutic effects to desired targets. The different types of nanocarriers include polymeric, viral, lipidic, metal-based (including a specific subclass - magnetic), and carbon-based.

While polymeric micelles and colloidal particles are more promising due to their high intrinsic biocompatibility and ability to easily penetrate the cellular membrane, metallic and CNMs show promise because they can provide more cancer-killing mechanisms, including PTT, PDT, and allow for direct visualization, enabling a theranostic approach. VPNs, and their specific subclass, OVs, on the other hand, are a completely different class of NMs, which can easily enter the cancer cells where they can deliver their cargo and/or transfect the cells for efficient tumor eradication, combined with immunity development.

Certainly, one of the main challenges nowadays in designing and successfully employing the novel NMs systems for fighting chemoresistant cancer is to guarantee high cancer-killing efficiency while still maintaining satisfactory systemic biocompatibility of the system in vivo. A literature survey presented herein seems to be pointing out that these goals cannot be achieved by using simple, two- or threecomponent systems. Specifically, a simple drug carrier strategy does not seem to be sufficiently effective. This is because chemoresistant and metastatic cancer is very hard to kill and the remission rate is significant. Hence, one does not only need for the NM-system to selectively target the cancer cells but also there are needs for the system to allow for the employment of additional cancer-killing mechanisms, such as PDT or PTT which can boost the therapeutic effect, additionally sensitizing the cells. Preferably, such a system should also be combined with an ability to directly visualize the cancer cells inside the body for simple, high-resolution tracking of treatment efficiency and cancer response. This approach allows for realtime diagnosis, creating an ability for an alternate treatment as needed. 

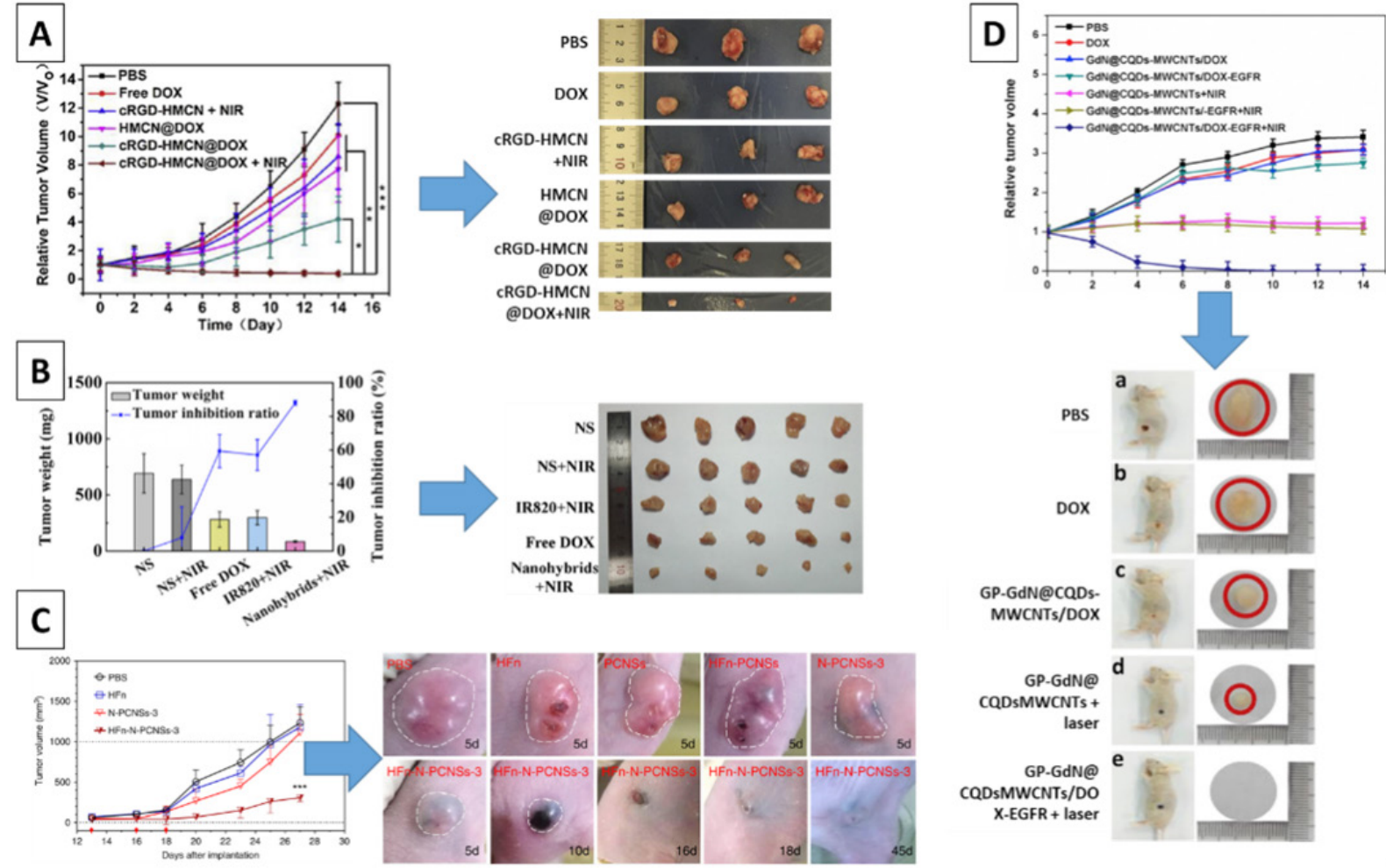

Figure 10. Examples of efficient targeting of tumors in vivo, with the aid of CNMs. Size reduction (A and B) and complete tumor eradication (C and D) can be observed. The materials are $(A)$ carbon nanoparticles ${ }^{[135]},(B) \mathrm{GO}^{[183]},(C)$ carbon nanozyme ${ }^{[139]}$, and $(D)$ $\mathrm{CNTS}^{[191]}$

These goals can be achieved through multiple routes, the simplest idea being polymeric and lipid carriers, filled with metallic or carbon nanoparticles and covered/embedded with specific cancer-targeting molecules. Such a carrier may also contain a drug and/or VPN. However, while the idea is simple, the complexity of the fabrication procedure, a risk of constituent chemical or physical cross-interactions, which can reduce their effectiveness, combined with the relatively large size of the system, significantly limits the practical employment of this approach. For these reasons, utilizing the metal and carbon-based NMs' intrinsic properties seems to be more promising. This approach needs employing strict fabrication and handling procedures, combined with extensive physicochemical analysis of the carrier, which only then would allow for the obtainment of safe and biocompatible systems. Further functionalization techniques can be tailored and employed to introduce strictly desired drug binding/cancer cell targeting mechanisms. Because these steps can be extremely time-consuming and expensive, they are rarely properly employed, leading to large discrepancies and skepticism of the scientific community in CNMs and metallic particles biomedical applications. Still, as the literature survey suggests, upon careful selection, these NMs can yield results unparalleled with other systems, creating multiple drug binding options, being effective PDT and PTT systems, and allowing for a theranostic approach due to their magnetic and/or fluorescence properties.

Overall, metal-based nanoparticles and carbon-based nanomaterials present extremely viable options for cancer-treatment because of their biocompatibility and stability. However, other nanomaterials are being explored in research that likely will eventually produce highly-valuable therapeutics.

\section{DECLARATIONS}

\section{Authors' contributions}

Conceptualized the content of this work: Medina-Cruz D, Webster TJ

Performed the literature review and writing, and composed the multipanel images: Benko A, Medina-Cruz 
D, Vernet-Crua A, O’Connell CP, Świętek M, Barabadi H, Saravanan M

Prepared the original images for the manuscript, responsible for final editing: Benko A

Completed the proofreading of the original article: Benko A, Medina-Cruz D, Vernet-Crua A

Performed the revision and proofreading of the revised article, and corresponded with the reviewers:

Benko A, Medina-Cruz D

Corresponded with the editorial office: Medina-Cruz D

\section{Availability of data and materials}

All data sources could be available to readers on request.

\section{Financial support and sponsorship}

Benko A is supported by a grant from the National Science Center, Poland (UMO-2017/24/C/ST8/00400), Świętek M is supported by the NPU II (LQ1604) provided by MEYS, and CZ.1.05/1.1.00/02.0109 BIOCEV provided by ERDF and MEYS.

\section{Conflicts of interest}

All authors declared that there are no conflicts of interest.

\section{Ethical approval and consent to participate}

Not applicable.

\section{Consent for publication}

Not applicable.

\section{Copyright}

(c) The Author(s) 2021.

\section{REFERENCES}

1. Sarkar S, Horn G, Moulton K, et al. Cancer development, progression, and therapy: an epigenetic overview. Int J Mol Sci 2013; 14:21087113.

2. Siegel RL, Miller KD, Jemal A. Cancer statistics, 2020. CA Cancer J Clin 2020;70:7-30.

3. Greenivald P, Dunn BK. Landmarks in the history of cancer epidemiology. Cancer Res 2009;69:2151-62.

4. Van der Meel R, Sulheim E, Shi Y, et al. Smart cancer nanomedicine. Nat Nanotechnol 2019;14:1007-17.

5. Chen H, Zhang W, Zhu G, Xie J, Chen X. Rethinking cancer nanotheranostics. Nat Rev Mater 2017;2:1-18.

6. Lippert TH, Ruoff HJ, Volm M. Intrinsic and acquired drug resistance in malignant tumors: The main reason for therapeutic failure. Arzneimittelforschung 2008;58:261-4.

7. Housman G, Byler S, Heerboth S, et al. Drug resistance in cancer: an overview. Cancers (Basel) 2014;6:1769-92.

8. Gottesman MM. Mechanisms of cancer drug resistance. Annu Rev Med 2002;53:615-27.

9. Yang J, Yu Y, Liu W, et al. Microtubule-associated protein tau is associated with the resistance to docetaxel in prostate cancer cell lines. Res Rep Urol 2017;9:71-7.

10. Pan ST, Li ZL, He ZX, Qiu JX, Zhou SF. Molecular mechanisms for tumour resistance to chemotherapy. Clin Exp Pharmacol Physiol 2016;43:723-37.

11. Kalal BS, Upadhya D, Pai VR. Chemotherapy resistance mechanisms in advanced skin cancer. Oncol Rev 2017;11:19-25.

12. Suzawa K, Offin M, Schoenfeld AJ, et al. Acquired MET Exon 14 alteration drives secondary resistance to epidermal growth factor receptor tyrosine kinase inhibitor in EGFR -mutated lung cancer. JCO Precis Oncol 2019;3:1-8.

13. Robey RW, Pluchino KM, Hall MD, et al. Revisiting the role of ABC transporters in multidrug-resistant cancer. Nat Rev Cancer 2018;18:452-64

14. Hermawan A, Wagner E, Roidl A. Consecutive salinomycin treatment reduces doxorubicin resistance of breast tumor cells by diminishing drug efflux pump expression and activity. Oncol Rep 2016;35:1732-40.

15. $\mathrm{Hu} \mathrm{T}, \mathrm{Li} \mathrm{Z}$, Gao CY, Cho CH. Mechanisms of drug resistance in colon cancer and its therapeutic strategies. World J Gastroenterol 2016;22:6876-89

16. Kim SJ, Kim S, Kim DW, et al. Alterations in PD-L1 expression associated with acquisition of resistance to ALK inhibitors in ALKrearranged lung cancer. Cancer Res Treat 2019;51:1231-40

17. Toth RK, Tran JD, Muldong MT, et al. Hypoxia-induced PIM kinase and laminin-activated integrin $\$ \alpha \$ 6$ mediate resistance to PI3K 
inhibitors in bone-metastatic CRPC. Am J Clin Exp Urol 2019;7:297-312.

18. Wang S, Liu F, Zhu J, et al. DNA repair genes ERCC1 and BRCA1 expression in non-small cell lung cancer chemotherapy drug resistance. Med Sci Monit 2016;22:1999-2005.

19. Nogales V, Reinhold WC, Varma S, et al. Epigenetic inactivation of the putative DNA/RNA helicase SLFN11 in human cancer confers resistance to platinum drugs. Oncotarget 2016;7:3084-97.

20. Dagogo-Jack I, Shaw AT. Tumour heterogeneity and resistance to cancer therapies. Nat Rev Clin Oncol 2018;15:81-94.

21. Russo M, Siravegna G, Blaszkowsky LS, et al. Tumor heterogeneity and Lesion-Specific response to targeted therapy in colorectal cancer. Cancer Discov 2016;6:147-53.

22. Mansoori B, Mohammadi A, Davudian S, Shirjang S, Baradaran B. The different mechanisms of cancer drug resistance: a brief review. Adv Pharm Bull 2017;7:339-48.

23. Du B, Shim J. Targeting epithelial-mesenchymal transition (EMT) to overcome drug resistance in cancer. Molecules 2016;21:965.

24. Elaskalani O, Razak NBA, Falasca M, Metharom P. Epithelial-mesenchymal transition as a therapeutic target for overcoming chemoresistance in pancreatic cancer. World J Gastrointestinal Oncol 2017;9:37-41.

25. Islam SU, Shehzad A, Sonn JK, Lee YS. PRPF overexpression induces drug resistance through actin cytoskeleton rearrangement and epithelial-mesenchymal transition. Oncotarget 2017;8:56659-71.

26. Gao M, Deng J, Liu F, et al. Triggered ferroptotic polymer micelles for reversing multidrug resistance to chemotherapy. Biomaterials 2019;223:119486.

27. Tran S, DeGiovanni PJ, Piel B, Rai P. Cancer nanomedicine: a review of recent success in drug delivery. Clin Transl Med 2017;6:44.

28. Xin Y, Yin M, Zhao L, Meng F, Luo L. Recent progress on nanoparticle-based drug delivery systems for cancer therapy. Cancer Biol Med 2017;14:228-41.

29. Truong NP, Whittaker MR, Mak CW, Davis TP. The importance of nanoparticle shape in cancer drug delivery. Expert Opin Drug Del 2015;12:129-42

30. Li Z, Tan S, Li S, Shen Q, Wang K. Cancer drug delivery in the nano era: an overview and perspectives (Review). Oncol Rep 2017;38:611-24.

31. Din FU, Aman W, Ullah I, et al. Effective use of nanocarriers as drug delivery systems for the treatment of selected tumors. Int $J$ Nanomedicine 2017;12:7291-309.

32. Kesharwani SS, Kaur S, Tummala H, Sangamwar AT. Multifunctional approaches utilizing polymeric micelles to circumvent multidrug resistant tumors. Colloids Surfaces B Biointerfaces 2019;173:581-90.

33. Raveendran R. Chapter 12 - Polymeric micelles: Smart nanocarriers for anticancer drug delivery. In: Sharma CP, editor. Drug Delivery Nanosystems for Biomedical Applications. Elsevier; 2018. pp. 255-73.

34. Yang X, Lian K, Tan Y, et al. Selective uptake of chitosan polymeric micelles by circulating monocytes for enhanced tumor targeting. Carbohydr Polym 2020;229:115435.

35. Yao Q, Liu Y, Kou L, et al. Tumor-targeted drug delivery and sensitization by MMP2-responsive polymeric micelles. Nanomedicine 2019;19:71-80.

36. Zhen S, Yi X, Zhao Z, et al. Drug delivery micelles with efficient near-infrared photosensitizer for combined image-guided photodynamic therapy and chemotherapy of drug-resistant cancer. Biomaterials 2019;218:119330.

37. Ambekar RS, Choudhary M, Kandasubramanian B. Recent advances in dendrimer-based nanoplatform for cancer treatment: a review. Eur Polym J 2020;126:109546.

38. Choudhary S, Gupta L, Rani S, Dave K, Gupta U. Impact of dendrimers on solubility of hydrophobic drug molecules. Front Pharmacol 2017;8:261

39. Rajani C, Borisa P, Karanwad T, et al. 7 - Cancer-targeted chemotherapy: Emerging role of the folate anchored dendrimer as drug delivery nanocarrier. In: Chauhan A, Kulhari H, editors. Pharmaceutical Applications of Dendrimers. Elsevier; 2020. pp. 151-98.

40. Siriviriyanun A, Tsai YJ, Voon SH, et al. Cyclodextrin- and dendrimer-conjugated graphene oxide as a nanocarrier for the delivery of selected chemotherapeutic and photosensitizing agents. Mater Sci Eng C 2018;89:307-15.

41. Golshan M, Salami-Kalajahi M, Mirshekarpour M, Roghani-Mamaqani H, Mohammadi M. Synthesis and characterization of poly(propylene imine)-dendrimer-grafted gold nanoparticles as nanocarriers of doxorubicin. Colloids Surfaces B Biointerfaces 2017; 155:257-65.

42. Fan Y, Yuan S, Huo M, et al. Spatial controlled multistage nanocarriers through hybridization of dendrimers and gelatin nanoparticles for deep penetration and therapy into tumor tissue. Nanomedicine 2017;13:1399-410.

43. Rompicharla SVK, Kumari P, Bhatt H, Ghosh B, Biswas S. Biotin functionalized PEGylated poly(amidoamine) dendrimer conjugate for active targeting of paclitaxel in cancer. Int J Pharm 2019;557:329-41.

44. Liang S, Sun C, Yang P, et al. Core-shell structured upconversion nanocrystal-dendrimer composite as a carrier for mitochondria targeting and catalase enhanced anti-cancer photodynamic therapy. Biomaterials 2020;240:119850.

45. Pan J, Mendes LP, Yao M, et al. Polyamidoamine dendrimers-based nanomedicine for combination therapy with siRNA and chemotherapeutics to overcome multidrug resistance. Eur J Pharm Biopharm 2019;136:18-28.

46. Gouveia M, Figueira J, Jardim MG, et al. Poly(alkylidenimine) dendrimers functionalized with the organometallicmoiety [Ru(v 5-C5H5) (PPh3)2]+ as promising drugs against cisplatin-resistant cancer cells and humanmesenchymal stem cells. Molecules 2018;23:1471.

47. Messager L, Gaitzsch J, Chierico L, Battaglia G. Novel aspects of encapsulation and delivery using polymersomes. Curr Opin Pharmacol 2014;18:104-11.

48. Dan N. Chapter 1 - vesicle-based drug carriers: liposomes, polymersomes, and niosomes. In: Grumezescu AM, editor. Design and 
Development of New Nanocarriers. William Andrew Publishing; 2018. pp. 1-55.

49. Khan MA, Ali S, Venkatraman SS, et al. Fabrication of poly (butadiene-block-ethylene oxide) based amphiphilic polymersomes: an approach for improved oral pharmacokinetics of Sorafenib. Int J Pharm 2018;542:196-204.

50. Köthe T, Martin S, Reich G, Fricker G. Dual asymmetric centrifugation as a novel method to prepare highly concentrated dispersions of PEG-b-PCL polymersomes as drug carriers. Int J Pharm 2020;579:119087.

51. Liu Q, Song L, Chen S, et al. A superparamagnetic polymersome with extremely high T2 relaxivity for MRI and cancer-targeted drug delivery. Biomaterials 2017;114:23-33.

52. Zhu D, Wu S, Hu C, et al. Folate-targeted polymersomes loaded with both paclitaxel and doxorubicin for the combination chemotherapy of hepatocellular carcinoma. Acta Biomater 2017;58:399-412.

53. Zavvar T, Babaei M, Abnous K, et al. Synthesis of multimodal polymersomes for targeted drug delivery and MR/fluorescence imaging in metastatic breast cancer model. Int J Pharm 2020;578:119091.

54. Simón-Gracia L, Hunt H, Scodeller PD, et al. Paclitaxel-loaded polymersomes for enhanced intraperitoneal chemotherapy. Mol Cancer Ther 2016;15:670.

55. Alibolandi M, Ramezani M, Abnous K, Hadizadeh F. AS1411 aptamer-decorated biodegradable polyethylene glycol-poly(lacticco-glycolic acid) nanopolymersomes for the targeted delivery of gemcitabine to non-small cell lung cancer in vitro. $J$ Pharm Sci 2016;105:1741-50.

56. Alibolandi M, Abnous K, Hadizadeh F, et al. Dextran-poly lactide-co-glycolide polymersomes decorated with folate-antennae for targeted delivery of docetaxel to breast adenocarcinima in vitro and in vivo. $J$ Control Release 2016;241:45-56.

57. Qin Y, Zhang Z, Huang C, et al. Folate-targeted redox-responsive polymersomes loaded with chemotherapeutic drugs and tariquidar to overcome drug resistance. J Biomed Nanotechnol 2018;14:1705-18.

58. Franke CE, Czapar AE, Patel RB, Steinmetz NF. Tobacco mosaic virus-delivered cisplatin restores efficacy in platinum-resistant ovarian cancer cells. Mol Pharm 2018;15:2922-31.

59. Perillo E, Porto S, Falanga A, et al. Liposome armed with herpes virus-derived gH625 peptide to overcome doxorubicin resistance in lung adenocarcinoma cell lines. Oncotarget 2016;7:4077-92.

60. Bell J, McFadden G. Viruses for tumor therapy. Cell Host Microbe 2014;15:260-5.

61. Hou W, Sampath P, Rojas JJ, Thorne SH. Oncolytic virus-mediated targeting of PGE2 in the tumor alters the immune status and sensitizes established and resistant tumors to immunotherapy. Cancer Cell 2016;30:108-19.

62. Mahoney DJ, Lefebvre C, Allan K, et al. Virus-Tumor interactome screen reveals ER stress response can reprogram resistant cancers for oncolytic virus-triggered caspase-2 cell death. Cancer Cell 2011;20:443-56.

63. Muscolini M, Castiello L, Palermo E, et al. SIRT1 modulates the sensitivity of prostate cancer cells to vesicular stomatitis virus oncolysis. J Virol 2019;93:e00626-19.

64. Dold C, Rodriguez Urbiola C, Wollmann G, et al. Application of interferon modulators to overcome partial resistance of human ovarian cancers to VSV-GP oncolytic viral therapy. Mol Ther Oncolytics 2016;3:16021.

65. Martikainen M, Niittykoski M, von und zu Fraunberg M, et al. MicroRNA-attenuated clone of virulent semliki forest virus overcomes antiviral type i interferon in resistant mouse CT-2A glioma. $J$ Virol 2015;89:10637-47.

66. Subramani T, Ganapathyswamy H. An overview of liposomal nano-encapsulation techniques and its applications in food and nutraceutical. J Food Sci Technol 2020;57:3545-55.

67. Mehta PP, Ghoshal D, Pawar AP, Kadam SS, Dhapte-Pawar VS. Recent advances in inhalable liposomes for treatment of pulmonary diseases: concept to clinical stance. J Drug Deliv Sci Technol 2020;56:101509.

68. Hossen S, Hossain MK, Basher MK, et al. Smart nanocarrier-based drug delivery systems for cancer therapy and toxicity studies: a review. J Adv Res 2019;15:1-18

69. Jampílek J, Král'ová K. Chapter 8 - recent advances in lipid nanocarriers applicable in the fight against cancer. In: Grumezescu AM, editor. Nanoarchitectonics in Biomedicine. William Andrew Publishing; 2019. pp. 219-94.

70. Chauhan SB, Gupta V. Recent advances in liposome. Res J Pharm Technol 2020;13:2053-8.

71. Kiaie N, Gorabi AM, Penson PE, et al. A new approach to the diagnosis and treatment of atherosclerosis: the era of the liposome. Drug Discov Today 2020;25:58-72.

72. Sercombe L, Veerati T, Moheimani F, et al. Advances and challenges of liposome assisted drug delivery. Front Pharmacol 2015;6:286.

73. Crommelin DJA, van Hoogevest P, Storm G. The role of liposomes in clinical nanomedicine development. What now? Now what? $J$ Control Release 2020;318:256-63.

74. Paliwal SR, Paliwal R, Agrawal GP, Vyas SP. Hyaluronic acid modified pH-sensitive liposomes for targeted intracellular delivery of doxorubicin. J Liposome Res 2016;26:276-87.

75. Chen M, Song F, Liu Y, et al. A dual pH-sensitive liposomal system with charge-reversal and NO generation for overcoming multidrug resistance in cancer. Nanoscale 2019;11:3814-26.

76. Feng X, Li L, Jiang H, et al. Dihydroartemisinin potentiates the anticancer effect of cisplatin via mTOR inhibition in cisplatin-resistant ovarian cancer cells: involvement of apoptosis and autophagy. Biochem Biophys Res Commun 2014;444:376-81.

77. Qiu L, Gao M, Xu Y. Enhanced combination therapy effect on paclitaxel-resistant carcinoma by chloroquine co-delivery via liposomes. Int J Nanomedicine 2015;10:6615.

78. Kang XJ, Wang HY, Peng HG, et al. Codelivery of dihydroartemisinin and doxorubicin in mannosylated liposomes for drug-resistant colon cancer therapy. Acta Pharmacol Sin 2017;38:885-96.

79. Li N, Mai Y, Liu Q, Gou G, Yang J. Docetaxel-loaded D- $\alpha$-tocopheryl polyethylene glycol-1000 succinate liposomes improve lung cancer 
chemotherapy and reverse multidrug resistance. Drug Deliv Transl Res 2020; doi: 10.1007/s13346-020-00720-9.

80. Shen Q, Shen Y, Jin F, Du Y, Ying X. Paclitaxel/hydroxypropyl- $\beta$-cyclodextrin complex-loaded liposomes for overcoming multidrug resistance in cancer chemotherapy. J Liposome Res 2020;30:12-20.

81. Li X, Wu X, Yang H, et al. A nuclear targeted Dox-aptamer loaded liposome delivery platform for the circumvention of drug resistance in breast cancer. Biomed Pharmacother 2019;117:109072.

82. Nasirizadeh S, Malaekeh-Nikouei B. Solid lipid nanoparticles and nanostructured lipid carriers in oral cancer drug delivery. J Drug Deliv Sci Technol 2020;55:101458.

83. Bayón-Cordero L, Alkorta I, Arana L. Application of solid lipid nanoparticles to improve the efficiency of anticancer drugs. Nanomaterials 2019;9:474.

84. Abdelaziz HM, Freag MS, Elzoghby AO. Chapter 5 - solid lipid nanoparticle-based drug delivery for lung cancer. In: Kesharwani P, editor. Nanotechnology-Based Targeted Drug Delivery Systems for Lung Cancer. Academic Press; 2019. pp. 95-121.

85. Rajabi M, Mousa SA. Lipid nanoparticles and their application in nanomedicine. Curr Pharm Biotechnol 2016;17:662-72.

86. Mihai MM, Holban AM, Călugăreanu A, Orzan OA. Chapter 11 - Recent advances in diagnosis and therapy of skin cancers through nanotechnological approaches. In: Ficai A, Grumezescu AM, editors. Nanostructures for Cancer Therapy. Elsevier; 2017. pp. 285-306.

87. Trapani A, Mandracchia D, Tripodo G, et al. Solid lipid nanoparticles made of self-emulsifying lipids for efficient encapsulation of hydrophilic substances. AIP Conference Proceedings 2145, 20004. AIP Publishing LLC; 2019.

88. Dumont C, Bourgeois S, Fessi H, Dugas PY, Jannin V. In-vitro evaluation of solid lipid nanoparticles: Ability to encapsulate, release and ensure effective protection of peptides in the gastrointestinal tract. Int J Pharm 2019;565:409-18.

89. Oner E, Kotmakci M, Kantarci AG. A promising approach to develop nanostructured lipid carriers from solid lipid nanoparticles: preparation, characterization, cytotoxicity and nucleic acid binding ability. Pharm Dev Technol 2020;25:936-48.

90. Rajpoot K, Jain SK. Oral delivery of pH-responsive alginate microbeads incorporating folic acid-grafted solid lipid nanoparticles exhibits enhanced targeting effect against colorectal cancer: a dual-targeted approach. Int J Biol Macromol 2020;151:830-44

91. Das Gupta S, Suh N. Tocopherols in cancer: an update. Mol Nutr Food Res 2016;60:1354-63.

92. Affram KO, Smith T, Ofori E, et al. Cytotoxic effects of gemcitabine-loaded solid lipid nanoparticles in pancreatic cancer cells. J Drug Deliv Sci Technol 2020;55:101374.

93. Oliveira MS, Aryasomayajula B, Pattni B, et al. Solid lipid nanoparticles co-loaded with doxorubicin and $\alpha$-tocopherol succinate are effective against drug-resistant cancer cells in monolayer and 3-D spheroid cancer cell models. Int J Pharm 2016;512:292-300.

94. Jiang T, Zhang C, Sun W, et al. Doxorubicin encapsulated in TPGS-modified 2D-nanodisks overcomes multidrug resistance. Chem A Eur $J$ 2020;26:2470-7.

95. Tang J, Ji H, Ren J, et al. Solid lipid nanoparticles with TPGS and brij 78: a co-delivery vehicle of cur and piperine for reversing P-Glycoprotein-Mediated multidrug resistance in vitro. Oncol Lett 2017;13:389-95.

96. Garg NK, Singh B, Jain A, et al. Fucose decorated solid-lipid nanocarriers mediate efficient delivery of methotrexate in breast cancer therapeutics. Colloids Surf B Biointerfaces 2016;146:114-26.

97. Wang F, Li L, Liu B, Chen Z, Li C. Hyaluronic acid decorated pluronic P85 solid lipid nanoparticles as a potential carrier to overcome multidrug resistance in cervical and breast cancer. Biomed Pharmacother 2017;86:595-604.

98. Zheng G, Zheng M, Yang B, Fu H, Li Y. Improving breast cancer therapy using doxorubicin loaded solid lipid nanoparticles: synthesis of a novel arginine-glycine-aspartic tripeptide conjugated, $\mathrm{pH}$ sensitive lipid and evaluation of the nanomedicine in vitro and in vivo. Biomed Pharmacother 2019;116:109006.

99. Pedrosa P, Corvo ML, Ferreira-Silva M, et al. Targeting cancer resistance via multifunctional gold nanoparticles. Int $J$ Mol Sci 2019;20:5510.

100. Rathinaraj P, Muthusamy G, Prasad NR, et al. Folate-gold-bilirubin nanoconjugate induces apoptotic death in multidrug-resistant oral carcinoma cells. Eur J Drug Metab Pharmacokinet 2020;45:285-96.

101. Kumon K, Kubota T, Kuroda S, et al. Abstract 3617: Trastuzumab-conjugated gold nanoparticles as novel HER2-targeted therapeutics against trastuzumab-resistant gastric cancer. Cancer Res 2019;79:3617.

102. Deng R, Ji B, Yu H, et al. Multifunctional gold nanoparticles overcome microRNA regulatory network mediated-multidrug resistant leukemia. Sci. Rep 2019;9:1-11.

103. Huai Y, Zhang Y, Xiong X, Das S, Bhattacharya R, Mukherjee P. Gold nanoparticles sensitize pancreatic cancer cells to gemcitabine. Cell Stress 2019;3:267-79.

104. Talamantez-Lyburn S, Brown P, Hondrogiannis N, et al. Gold nanoparticles loaded with cullin-5 DNA increase sensitivity to 17-AAG in cullin-5 deficient breast cancer cells. Int J Pharm 2019;564:281-92.

105. Gopisetty MK, Kovács D, Igaz N, et al. Endoplasmic reticulum stress: major player in size-dependent inhibition of P-glycoprotein by silver nanoparticles in multidrug-resistant breast cancer cells. J Nanobiotechnol 2019;17:9.

106. Ramezani T, Nabiuni M, Baharara J, Parivar K, Namvar F. Sensitization of resistance ovarian cancer cells to cisplatin by biogenic synthesized silver nanoparticles through p53 activation. Iran J Pharm Res 2019;18:222-31.

107. Kovács D, Szőke K, Igaz N, et al. Silver nanoparticles modulate ABC transporter activity and enhance chemotherapy in multidrug resistant cancer. Nanomedicine Nanotechnol Biol Med 2016;12:601-10.

108. Wang Y, Zhao R, Wang S, Liu Z, Tang R. In vivo dual-targeted chemotherapy of drug resistant cancer by rationally designed nanocarrier. Biomaterials 2016;75:71-81.

109. Fernández M, Javaid F, Chudasama V. Advances in targeting the folate receptor in the treatment/imaging of cancers. Chem Sci 2018;9:790-810. 
110. Cho MH, Kim S, Lee JH. et al. Magnetic tandem apoptosis for overcoming multidrug-resistant cancer. Nano Lett 2016;16:7455-60.

111. Truffi M, Colombo M, Sorrentino L, et al. Multivalent exposure of trastuzumab on iron oxide nanoparticles improves antitumor potential and reduces resistance in HER2-positive breast cancer cells. Sci Rep 2018;8:1-11.

112. Miller-Kleinhenz J, Guo X, Qian W, et al. Dual-targeting Wnt and uPA receptors using peptide conjugated ultra-small nanoparticle drug carriers inhibited cancer stem-cell phenotype in chemo-resistant breast cancer. Biomaterials 2018;152:47-62.

113. Liu E, Zhang M, Cui H, et al. Tat-functionalized Ag-Fe3O4 nano-composites as tissue-penetrating vehicles for tumor magnetic targeting and drug delivery. Acta Pharm Sin B 2018;8:956-68.

114. Weng H, Bejjanki NK, Zhang J, et al. TAT peptide-modified cisplatin-loaded iron oxide nanoparticles for reversing cisplatin-resistant nasopharyngeal carcinoma. Biochem Biophys Res Commun 2019;511:597-603.

115. Ma P, Xiao H, Yu C, et al. Enhanced cisplatin chemotherapy by iron oxide nanocarrier-mediated generation of highly toxic reactive oxygen species. Nano Lett 2017;17:928-37.

116. Guo S, Yao X, Jiang Q, et al. Dihydroartemisinin-loaded magnetic nanoparticles for enhanced chemodynamic therapy. Front Pharmacol 2020;11:1-11.

117. Yen TY, Stephen ZR, Lin G, et al. Catalase-functionalized iron oxide nanoparticles reverse hypoxia-induced chemotherapeutic resistance. Adv Healthc Mater 2019;8:1-8.

118. Roleira FM, Tavares-da-Silva EJ, Varela CL, et al. Plant derived and dietary phenolic antioxidants: anticancer properties. Food Chem 2015;183:235-58.

119. Wang J, Wang F, Li F, et al. A multifunctional poly(curcumin) nanomedicine for dual-modal targeted delivery, intracellular responsive release, dual-drug treatment and imaging of multidrug resistant cancer cells. J Mater Chem B 2016;4:2954-62.

120. Keskin T, Yalcin S., Gunduz U. Folic acid functionalized PEG coated magnetic nanoparticles for targeting anti-cancer drug delivery: preparation, characterization and cytotoxicity on Doxorubicin, Zoledronic acid and Paclitaxel resistant MCF-7 breast cancer cell lines. Inorg Nano-Metal Chem 2018;48:150-9.

121. Song W, Su X, Gregory DA, Li W, Cai Z, Zhao X. Magnetic alginate/chitosan nanoparticles for targeted delivery of curcumin into human breast cancer cells. Nanomaterials 2018;8:907.

122. Chen S, Liang Q, Liu E, et al. Curcumin/sunitinib co-loaded BSA-stabilized SPIOs for synergistic combination therapy for breast cancer. J Mater Chem B 2017;5:4060-72.

123. Rastegar R, Javar HA, Khoobi M, et al. Evaluation of a novel biocompatible magnetic nanomedicine based on beta-cyclodextrin, loaded doxorubicin-curcumin for overcoming chemoresistance in breast cancer. Artif Cells Nanomedicine Biotechnol 2018;46:207-16.

124. Daglioglu C. Enhancing tumor cell response to multidrug resistance with ph-sensitive quercetin and doxorubicin conjugated multifunctional nanoparticles. Colloids Surfaces B Biointerfaces 2017;156:175-85.

125. Wang D, Li X, Li X, et al. Magnetic and $\mathrm{pH}$ dual-responsive nanoparticles for synergistic drug-resistant breast cancer chemo/ photodynamic therapy. Int J Nanomedicine 2019;14:7665-79.

126. Shenderova OA, Hu Z, Brenner D. Carbon family at the nanoscale BT - synthesis, properties and applications of ultrananocrystalline diamond. In: Gruen DM, Shenderova OA, Vul AY, editors. Netherlands: Springer; 2005. pp. 1-14.

127. Heimann RB, Evsvukov SE, Koga Y. Carbon allotropes: a suggested classification scheme based on valence orbital hybridization. Carbon N Y 1997;35:1654-8.

128. Li D, Lin L, Fan Y, et al. Ultrasound-enhanced fluorescence imaging and chemotherapy of multidrug-resistant tumors using multifunctional dendrimer/carbon dot nanohybrids. Bioact 2020;6:729-39.

129. Li D, Fan Y, Shen M, Bányai I, Shi X. Design of dual drug-loaded dendrimer/carbon dot nanohybrids for fluorescence imaging and enhanced chemotherapy of cancer cells. J Mater Chem B 2019;7:277-85.

130. Patel KD, Singh RK, Kim HW. Carbon-based nanomaterials as an emerging platform for theranostics. Mater Horizons 2019;6:434-69.

131. Mehra NK, Palakurthi S. Interactions between carbon nanotubes and bioactives: a drug delivery perspective. Drug Discov Today 2016;21:585-97.

132. Maiti D, Tong X, Mou X, Yang K. Carbon-based nanomaterials for biomedical applications: a recent study. Front Pharmacol 2019;9:1401.

133. Bianco A, Pantarotto D, Kostarelos K, Prato M. Non-covalent complexes comprising carbon nanotubes. 2010. Available from: https:// patents.google.com/patent/US7858648. [Last accessed on 18 Nov 2020]

134. Iannazzo D, Pistone A, Celesti C, et al. A smart nanovector for cancer targeted drug delivery based on graphene quantum dots. Nanomaterials 2019;9:282.

135. Tian L, Tao L, Li H, et al. Hollow mesoporous carbon modified with cRGD peptide nanoplatform for targeted drug delivery and chemophotothermal therapy of prostatic carcinoma. Colloids Surfaces A Physicochem Eng Asp 2019;570:386-95.

136. Mahajan S, Patharkar A, Kuche K, et al. Functionalized carbon nanotubes as emerging delivery system for the treatment of cancer. Int $J$ Pharm 2018;548:540-58.

137. Loh KP, Ho D, Chiu GNC, et al. Clinical applications of carbon nanomaterials in diagnostics and therapy. Adv Mater 2018;30:1802368.

138. Taghavi S, Nia AH, Abnous K, Ramezani M. Polyethylenimine-functionalized carbon nanotubes tagged with AS1411 aptamer for combination gene and drug delivery into human gastric cancer cells. Int J Pharm 2017;516:301-12.

139. Fan K, Xi J, Fan L, et al. In vivo guiding nitrogen-doped carbon nanozyme for tumor catalytic therapy. Nat Commun 2018;9:1440.

140. Fan K, Cao C, Pan Y, et al. Magnetoferritin nanoparticles for targeting and visualizing tumour tissues. Nat. Nanotechnol 2012;7:459-64.

141. Alexander A, Agrawal M, Yadav P, et al. Chapter 17 - Targeted delivery through carbon nanomaterials: applications in bioactive delivery systems Edited by Singh MR, Singh D, Kanwar JR, Chauhan NSBT-A and A in the D of NC for B and BA. Academic Press; 2020. pp. 
$509-24$.

142. Pei X, Zhu Z, Gan Z, et al. PEGylated nano-graphene oxide as a nanocarrier for delivering mixed anticancer drugs to improve anticancer activity. Sci Rep 2020;10:1-15.

143. Qian R, Maiti D, Zhong J, et al. Multifunctional nano-graphene based nanocomposites for multimodal imaging guided combined radioisotope therapy and chemotherapy. Carbon N Y 2019;149:55-62.

144. Hong G, Diao S, Antaris AL, Dai H. Carbon nanomaterials for biological imaging and nanomedicinal therapy. Chem Rev 2015;115:10816906.

145. Costa PM, Bourgognon M, Wang JTW, Al-Jamal KT. Functionalized carbon nanotubes: From intracellular uptake and cell-related toxicity to systemic brain delivery. J Control Release 2016;241:200-19.

146. Kim SW, Lee YK, Kim SH, et al. Covalent, non-covalent, encapsulated nanodrug regulate the fate of intra- and extracellular trafficking: impact on cancer and normal cells. Sci Rep 2017;7:6454.

147. Ali MS, Metwally AA, Fahmy RH, Osman R. Nanodiamonds: minuscule gems that ferry antineoplastic drugs to resistant tumors. Int $J$ Pharm 2019;558:165-76.

148. Curcio M, Farfalla A, Saletta F, et al. Functionalized carbon nanostructures versus drug resistance: promising scenarios in cancer treatment. Molecules 2020;25:2102.

149. Hosnedlova B, Kepinska M, Fernandez C, et al. Carbon nanomaterials for targeted cancer therapy drugs: a critical review. Chem Rec 2019;19:502-22.

150. Mehra NK, Jain AK, Nahar M. Carbon nanomaterials in oncology: an expanding horizon. Drug Discov Today 2018;23:1016-25.

151. de Melo-Diogo D, Lima-Sousa R, Alves CG, Costa EC, Louro RO, Correia IJ. Functionalization of graphene family nanomaterials for application in cancer therapy. Colloids Surf B Biointerfaces 2018;171:260-75.

152. Liu J, Dong J, Zhang T, Peng Q. Graphene-based nanomaterials and their potentials in advanced drug delivery and cancer therapy. $J$ Control Release 2018;286:64-73.

153. Jiang B, Zhou B, Lin Z, Liang H, Shen X. Recent advances in carbon nanomaterials for cancer phototherapy. Chem A Eur J 2019;25:3993-4004.

154. Mohajeri M, Behnam B, Sahebkar A. Biomedical applications of carbon nanomaterials: Drug and gene delivery potentials. J. Cell Physiol 2019;234:298-319.

155. Chen D, Dougherty CA, Zhu K, Hong H. Theranostic applications of carbon nanomaterials in cancer: focus on imaging and cargo delivery. J Control Release 2015;210:230-45.

156. Dong X, Sun Z, Wang X, Leng X. An innovative MWCNTs/DOX/TC nanosystem for chemo-photothermal combination therapy of cancer. Nanomed Nanotechnol Biol Med 2017;13:2271-80.

157. Meng Y, Wang S, Li C, et al. Photothermal combined gene therapy achieved by polyethyleneimine-grafted oxidized mesoporous carbon nanospheres. Biomaterials 2016;100:134-42.

158. Mohapatra S, Rout SR, Das RK, Nayak S, Ghosh SK. Highly hydrophilic luminescent magnetic mesoporous carbon nanospheres for controlled release of anticancer drug and multimodal imaging. Langmuir 2016;32:1611-20.

159. Zhao N, Fan W, Zhao X, et al. Polycation-carbon nanohybrids with superior rough hollow morphology for the NIR-II responsive multimodal therapy. ACS Appl Mater Interfaces 2020;12:11341-52.

160. Wang K, Yao H, Meng Y, et al. Specific aptamer-conjugated mesoporous silica-carbon nanoparticles for HER2-targeted chemophotothermal combined therapy. Acta Biomater 2015;16:196-205.

161. Li F, Wang Y, Zhang Z, Shen Y, Guo S. A chemo/photo- co-therapeutic system for enhanced multidrug resistant cancer treatment using multifunctional mesoporous carbon nanoparticles coated with poly (curcumin-dithiodipropionic acid). Carbon N Y 2017;122:524-37.

162. Tu X, Wang L, Cao Y, et al. Efficient cancer ablation by combined photothermal and enhanced chemo-therapy based on carbon nanoparticles/doxorubicin@SiO2 nanocomposites. Carbon N Y 2016;97:35-44.

163. Feng T, Ai X, An G, Yang P, Zhao Y. Charge-convertible carbon dots for imaging-guided drug delivery with enhanced in vivo cancer therapeutic efficiency. ACS Nano 2016;10:4410-20.

164. Feng T, Chua HJ, Zhao Y. Carbon-dot-mediated co-administration of chemotherapeutic agents for reversing cisplatin resistance in cancer therapy. ChemNanoMat 2018;4:801-6.

165. Ren W, Chen S, Liao Y, et al. Near-infrared fluorescent carbon dots encapsulated liposomes as multifunctional nano-carrier and tracer of the anticancer agent cinobufagin in vivo and in vitro. Colloids Surfaces B Biointerfaces 2019;174:384-92.

166. Chiu SH, Gedda G, Girma WM, et al. Rapid fabrication of carbon quantum dots as multifunctional nanovehicles for dual-modal targeted imaging and chemotherapy. Acta Biomater 2016;46:151-64.

167. Thakur M, Mewada A, Pandey S, et al. Milk-derived multi-fluorescent graphene quantum dot-based cancer theranostic system. Mater Sci Eng C 2016;67:468-77.

168. Sui X, Luo C, Wang C, et al. Graphene quantum dots enhance anticancer activity of cisplatin via increasing its cellular and nuclear uptake. Nanomed Nanotechnol Biol Med 2016;12:1997-2006.

169. Shenderova OA, Ciftan Hens SA. Nanodiamonds. Springer Handbook of Nanomaterials. Berlin Heidelberg: Springer; 2013. pp. 263-300.

170. Carbons and Nanocarbons BT - Nanomaterials for Solid State Hydrogen Storage. In: Varin RA, Czujko T, Wronski ZS, editors. US: Springer; 2009. pp. 291-320.

171. Yu Y, Yang X, Liu M, Nishikawa M, Tei T, Miyako E. Amphipathic nanodiamond supraparticles for anticancer drug loading and delivery. ACS Appl Mater Interfaces 2019;11:18978-87.

172. Zhu H, Wang Y, Hussain A, et al. Nanodiamond mediated co-delivery of doxorubicin and malaridine to maximize synergistic anti-tumor 
effects on multi-drug resistant MCF-7/ADR cells. J Mater Chem B 2017;5:3531-40.

173. Lam ATN, Yoon JH, Ly NH, Joo SW. Electrostatically self-assembled quinazoline-based anticancer drugs on negatively-charged nanodiamonds for overcoming the chemoresistances in lung cancer cells. Biochip J 2018;12:163-71.

174. Chan MS, Liu LS, Leung HM, Lo PK. Cancer-cell-specific mitochondria-targeted drug delivery by dual-ligand-functionalized nanodiamonds circumvent drug resistance. ACS Appl Mater Interfaces 2017;9:11780-9.

175. Li TF, Li K, Zhang Q, et al. Dendritic cell-mediated delivery of doxorubicin-polyglycerol-nanodiamond composites elicits enhanced anticancer immune response in glioblastoma. Biomaterials 2018;181:35-52.

176. Li TF, Xu YH, Li K, et al. Doxorubicin-polyglycerol-nanodiamond composites stimulate glioblastoma cell immunogenicity through activation of autophagy. Acta Biomater 2019;86:381-94.

177. Chen Z, Wang C, Li TF, et al. Doxorubicin conjugated with nanodiamonds and in free form commit glioblastoma cells to heterodromous fates. Nanomedicine 2019;14:335-51.

178. Chen Z, Yuan SJ, Li K, et al. Doxorubicin-polyglycerol-nanodiamond conjugates disrupt STAT3/IL-6-mediated reciprocal activation loop between glioblastoma cells and astrocytes. J Control Release 2020;320:469-83.

179. Yuan SJ, Xu YH, Wang C, et al. Doxorubicin-polyglycerol-nanodiamond conjugate is a cytostatic agent that evades chemoresistance and reverses cancer-induced immunosuppression in triple-negative breast cancer. J Nanobiotechnol 2019;17:110.

180. Tiwari H, Karki N, Pal M, et al. Functionalized graphene oxide as a nanocarrier for dual drug delivery applications: the synergistic effect of quercetin and gefitinib against ovarian cancer cells. Colloids Surf B Biointerfaces 2019;178:452-9.

181. Tran TH, Nguyen HT, Pham TT, et al. Development of a graphene oxide nanocarrier for dual-drug chemo-phototherapy to overcome drug resistance in cancer. ACS Appl Mater Interfaces 2015;7:28647-55.

182. Thapa RK, Youn YS, Jeong JH, Choi HG, Yong CS, Kim JO. Graphene oxide-wrapped PEGylated liquid crystalline nanoparticles for effective chemo-photothermal therapy of metastatic prostate cancer cells. Colloids Surf B Biointerfaces 2016;143:271-7.

183. Huang C, Hu X, Hou Z, Ji J, Li Z, Luan Y. Tailored graphene oxide-doxorubicin nanovehicles via near-infrared dye-lactobionic acid conjugates for chemo-photothermal therapy. J. Colloid Interface Sci 2019;545:172-83.

184. Han C, Zhang C, Ma T, et al. Hypericin-functionalized graphene oxide for enhanced mitochondria-targeting and synergistic anticancer effect. Acta Biomater 2018;77:268-81.

185. Guo L, Shi H, Wu H, et al. Prostate cancer targeted multifunctionalized graphene oxide for magnetic resonance imaging and drug delivery. Carbon N Y 2016;107:87-99.

186. Luo Y, Cai X, Li H, Lin Y, Du D. Hyaluronic acid-modified multifunctional Q-graphene for targeted killing of drug-resistant lung cancer cells. ACS Appl Mater Interfaces 2016;8:4048-55.

187. Chatterjee N, Yang J, Kim S, Joo SW, Choi J. Diameter size and aspect ratio as critical determinants of uptake, stress response, global metabolomics and epigenetic alterations in multi-wall carbon nanotubes. Carbon N Y 2016;108:529-40.

188. Donaldson K, Poland CA. Nanotoxicology: new insights into nanotubes. Nature Nanotechnol 2009;4:708-10.

189. Dong X, Sun Z, Wang X, et al. Simultaneous monitoring of the drug release and antitumor effect of a novel drug delivery systemMWCNTs/DOX/TC. Drug Deliv 2017;24:143-51.

190. Raza K, Kumar D, Kiran C, et al. Conjugation of docetaxel with multiwalled carbon nanotubes and codelivery with piperine: Implications on pharmacokinetic profile and anticancer activity. Mol Pharm 2016;13:2423-32.

191. Zhang M, Wang W, Wu F, Yuan P, Chi C, Zhou N. Magnetic and fluorescent carbon nanotubes for dual modal imaging and photothermal and chemo-therapy of cancer cells in living mice. Carbon N Y 2017;123:70-83.

192. Guven A, Rusakova IA, Lewis MT, Wilson LJ. Cisplatin@US-tube carbon nanocapsules for enhanced chemotherapeutic delivery. Biomaterials 2012;33:1455-61.

193. Guven A, Villares GJ, Hilsenbeck SG, et al. Carbon nanotube capsules enhance the in vivo efficacy of cisplatin. Acta Biomater 2017;58:466-78

194. Ghosh M, Das PK. Doxorubicin loaded 17ß-estradiol based SWNT dispersions for target specific killing of cancer cells. Colloids Surfaces B Biointerfaces 2016;142:367-76.

195. Razzazan A, Atyabi F, Kazemi B, Dinarvand R. In vivo drug delivery of gemcitabine with PEGylated single-walled carbon nanotubes. Mater Sci Eng C 2016; 62:614-25.

196. Li Y, Lu A, Long M, Cui L, Chen Z, Zhu L. Nitroimidazole derivative incorporated liposomes for hypoxia-triggered drug delivery and enhanced therapeutic efficacy in patient-derived tumor xenografts. Acta Biomater 2019;83:334-48. 